\title{
A Synoptic View of the Onset of the Midlatitude QBO Signal
}

\author{
Vered Silverman, ${ }^{a}$ Sandro W. Lubis, ${ }^{b}$ Nili Harnik, ${ }^{\mathrm{c}}$ And Katja Matthes ${ }^{\mathrm{d}}$ \\ ${ }^{\text {a }}$ Department of Environmental Sciences, Weizmann Institute, Rehovot, Israel \\ ${ }^{\mathrm{b}}$ Rice University, Houston, Texas \\ ${ }^{\mathrm{c}}$ School of the Environment and Earth Sciences, Tel Aviv University, Tel Aviv, Israel \\ ${ }^{\mathrm{d}}$ GEOMAR Helmholtz Centre for Ocean Research Kiel, Kiel, Germany
}

(Manuscript received 22 December 2020, in final form 5 August 2021)

\begin{abstract}
The extratropical effect of the quasi-biennial oscillation (QBO), known as the Holton-Tan effect, is manifest as a weaker, warmer winter Arctic polar vortex during the east QBO phase. While previous studies have shown that the extratropical QBO signal is caused by the modified propagation of planetary waves in the stratosphere, the mechanism dominating the onset and seasonal development of the Holton-Tan effects remains unclear. Here, the governing wave-mean flow dynamics of the early winter extratropical QBO signal onset and its reversibility is investigated on a synoptic time scale with a finite-amplitude diagnostic using reanalysis and a chemistry-climate model. The extratropical QBO signal onset in October is found to primarily result from modulated stratospheric life cycles of wave pulses entering the stratosphere from the troposphere, rather than from a modulation of their tropospheric wave source. A comprehensive analysis of the wave activity budget during fall, when the stratospheric winter polar vortex starts forming and waves start propagating up into the stratosphere, shows significant differences. During the east QBO phase, the deceleration of the mid-high-latitude stratospheric zonal-mean jet by the upward-propagating wave pulses is less reversible, due to stronger dissipation processes, while during the west phase, a more reversible deceleration of the main polar vortex is found owing to the waves being dissipated at lower latitudes, accompanied by a weak but different response of the tropospheric subtropical jet. From this synoptic wave-event viewpoint, the early season onset of the Holton-Tan effect results from the cumulative effect of the QBO dependent waveinduced deceleration during the life cycle of individual upward wave pulses.
\end{abstract}

SIGNIFICANCE STATEMENT: The quasi-biennial oscillation $(\mathrm{QBO})$ is the dominant signal in the tropical stratosphere, and its extratropical influence can potentially provide extended seasonal predictability. The midlatitude effect of the QBO discussed in the literature is by modulating upward-propagating planetary waves, focusing on the frequency of polar vortex breakup events (sudden stratospheric warmings) during midwinter. We focus on the overlooked onset of the early winter midlatitude QBO signal, showing a persistent QBO modulation of upward-propagating-wave life cycles. For this we use a diagnostic tailored explicitly to examine the contribution of nonconservative processes to wave-induced deceleration of the mean flow. The findings also provide a demonstration of how fundamental wave-mean flow concepts are manifest under realistic settings. This can aid in improving the midlatitude teleconnection of the QBO in climate simulations, as well as better understanding other processes that influence the midlatitude QBO teleconnection.

KEYWORDS: Planetary waves; Quasibiennial oscillation; Rossby waves; Stratospheric circulation; Teleconnections; Wave breaking; Eddies; Stratosphere-troposphere coupling; Stratosphere; Potential vorticity; General circulation models; Reanalysis data; Mixing; Synoptic-scale processes

\section{Introduction}

The quasi-biennial oscillation (QBO) is the tropical phenomenon of the zonal-mean winds alternating from easterly to westerly in the tropical latitudinal band $\left(\sim \pm 10^{\circ}\right)$, as the anomalies descend from the upper to lower stratosphere, with an average period of about 28 months. The influence of the QBO on the extratropical region has been referred to as the Holton-Tan effect, and it is manifested as a weaker and warmer winter Arctic polar vortex during the easterly phase of the QBO (cf. Holton and Tan 1980). The QBO itself results from the interaction between the zonal-mean zonal winds and upward-propagating tropical waves of opposing zonal phase speeds. To satisfy thermal wind balance, the QBO induced vertical wind shear induces an

Corresponding author: Vered Silverman, vered.silverman@ gmail.com overturning mean meridional circulation in the subtropics, with upwelling/downwelling in the lower tropical and upper subtropical stratosphere regions (Plumb and Bell 1982; Ruzmaikin et al. 2005; Garfinkel et al. 2012).

While the QBO is a tropical phenomenon, it has been noted by many studies that it influences higher latitudes. The first to notice this was Ebdon (1975), who found significantly higher polar surface pressure in January during east QBO. In attempt to explain this tropical-extratropical teleconnection, Holton and Tan (1980) analyzed 16 years of observational data. They found lower values of 50-hPa geopotential heights during east QBO, and attributed this to a modulation of the stratospheric waveguide by the more poleward position of the zero wind line during east QBO, which focuses the planetary wave activity to the polar vortex region.In addition to changing the position of the zero wind line, the QBOrelated acceleration/deceleration of the tropical zonal-mean zonal winds also induces an anomalous overturning circulation, which 
extends to the subtropics, where it induces further zonal-mean zonal wind anomalies, as well as temperature anomalies (Plumb and Bell 1982). Some studies have suggested that these secondary subtropical wind and temperature anomalies, and their corresponding influence on the index of refraction for extratropical planetary waves, is responsible for increased Eliassen-Palm (EP) flux convergence in the polar vortex region, and stronger wave deceleration in the east QBO phase (Ruzmaikin et al. 2005; Garfinkel et al. 2012; Hansen et al. 2013; Li and Tung 2014). White et al. (2016) further found that during east QBO there is increased upward stationary planetary wave propagation from the troposphere into the stratosphere, resulting from a combination of both the focusing of waves to the higher latitudes due to the change in location of the zero-wind line and the subtropical meridional circulation associated with the QBO winds modulating the index of refraction. They also showed that the east QBO phase exhibits significantly stronger nonlinear effects in the wave enstrophy budget. For a comprehensive review of the extratropical effect of the QBO see Dunkerton and Baldwin (1991) and Anstey and Shepherd (2014).

The Holton-Tan effect exhibits a seasonal cycle, and is in fact found to be more robust in early winter. This was shown in observational data (Naito and Hirota 1997), and using reanalysis data (Hu and Tung 2002; Gray et al. 2004; Lu et al. 2008; Anstey and Shepherd 2014). Despite this, the early winter onset of the Holton-Tan effect has received little attention, as most studies focused on the midwinter conditions or used monthly means to study the QBO effect on the propagation of planetary waves. Currently, some climate models are unable to produce the early onset of the Holton-Tan signal, so that it appears only later in winter (e.g., in December rather than in October; e.g., Watson and Gray 2014). In this study we focus on understanding the key processes responsible for initiating the extratropical QBO signal during fall.

The deceleration of the vortex by waves is episodic due to the intermittent nature of upward wave propagation. According to the generalized Eliassen-Palm theorem, an increase in wave activity with time will result in zonal-mean zonal wind deceleration, while a decrease in wave activity with time will lead to zonal-mean zonal wind acceleration (Andrews and McIntyre 1976). If a wave packet enters a region and leaves it with no dissipation, the deceleration at the leading edge of the wave packet as it enters the region will be fully reversed by the acceleration at its trailing edge when it leaves the region, with a zero net change in the mean flow. This can be thought of as the nonacceleration theorem (Charney and Drazin 1961), applied to the time mean over the wave life cycle. Thus, the net amount of deceleration of the midlatitude stratospheric polar vortex by a wave packet entering it from below depends on how much of its wave activity gets dissipated within the midlatitude vortex during its "life cycle." During fall (September-November), when there is no downward reflection of waves from the stratosphere back to the troposphere (Perlwitz and Harnik 2003), waves propagating up to the stratosphere from the troposphere will tend to get refracted to the subtropics where they are dissipated in a region referred to as the surf zone (Plumb 2002). Diagnostically, if we define the stratospheric polar vortex region as the region between the tropopause and stratopause, poleward of a latitude circle at its subtropical edge, an upward pulse of wave activity will decelerate the vortex as it enters the stratosphere. This deceleration will be irreversible if the waves break within the polar vortex region; however, if the wave activity gets refracted to the subtropics, the polar vortex will get accelerated back, while the subtropical zonal winds will be decelerated as the waves enter and break there. If net deceleration over the wave packet life cycle depends on how much of its wave activity gets dissipated within the vortex region, and how much leaves it via equatorward refraction ${ }^{1}$ by modulating the amounts of equatorward refraction of the upward wave pulses, the QBO can influence the net amount of wave-induced deceleration, and thus the polar vortex strength. Indeed, Silverman et al. (2018) showed that the wave-induced deceleration by upward-propagating waves in fall is almost fully reversible during west QBO, and much less reversible during east QBO. The cumulative effect of these differences is a stronger fall/early-winter polar vortex during the west QBO phase. Consistently, the nonlinear terms in the wave enstrophy budget were found to be significantly stronger during east QBO, similar to the findings of White et al. (2016).

The analysis done by Silverman et al. (2018) (and also in White et al. 2016), used the wave enstrophy budget to diagnose the evolution of wave activity. However, the enstrophy budget has a very large residual, making it hard to separate true irreversible nonlinear enstrophy mixing by nonconservative processes, and dissipation from numerical artifacts (e.g., due to the temporal resolution or the smallamplitude approximation, which exclude cubic terms from the enstrophy equation). These issues are particularly strong near zero wind lines, where the wave velocities are larger than the mean flow, or near regions where the zonalmean meridional potential vorticity gradient vanishes, and the wave activity [proportional to $\overline{q^{\prime 2}} /(d \bar{q} / d y)$, where $q$ is the potential vorticity, and the prime and the bar respectively denote deviations and averaging in the zonal direction] diverges. To overcome this limitation of the enstrophy budget analysis, we propose to use the Finite-Amplitude Wave Activity (FAWA) framework which was recently developed by Nakamura and Solomon (2010) as an extension of the small-amplitude wave activity (Andrews and McIntyre 1976). Instead of defining perturbations to be deviations from a zonal Eulerian mean, FAWA is calculated from the differences between an actual potential vorticity (PV) contour and its equivalent latitude. As a result, the FAWAbased derivation is valid for finite-amplitude perturbations, and it allows a much more accurate calculation of the contributions of diabatic processes and irreversible potential vorticity mixing, and correspondingly a better quantification of the reversibility of transient wave forcing during individual wave life cycles. We chose this framework over the impulse-Casimir-based wave activity (Killworth and McIntyre 1985; McIntyre and Shepherd 1987; Haynes 1988)

\footnotetext{
${ }^{1}$ Later in the season, downward reflection of wave activity (e.g. Perlwitz and Harnik 2003) can also lead to acceleration and a reversible net effect of the waves on the polar vortex (Harnik 2009; Shaw and Perlwitz 2013; Lubis et al. 2017).
} 
since the latter does not satisfy a nonacceleration theorem, due to an additional advection term coming from its Eulerian nature (Huang and Nakamura 2016).

In this study we investigate the seasonal development of the midlatitude QBO signal, using the FAWA framework to quantify the role of wave transience at finite amplitude and the nonconservative processes on the seasonal evolution of eddy forcing during east and west QBO. We establish a comprehensive analysis of the wave activity and wave-mean flow interaction budget to explain what makes the west QBO events more reversible, in reanalysis and a chemistry climate model. The paper is constructed as follows: First, the contribution of adiabatic and nonconservative processes in the climatological seasonal cycle are evaluated. Then upwardpropagating wave events during fall, when the polar vortex just gets established, are studied using the FAWA diagnostics. In particular, to quantify the reversibility of the wave induced deceleration during east/west QBO, the concept of the nonacceleration theorem is extended to a wave life cycle perspective. The main results are shown for the Japanese 55-year Reanalysis (JRA-55; 58 years), while additional results from a simulation of the Whole Atmosphere Community Climate Model (WACCM).

\section{Data and diagnostics}

Our analysis comprises examining the FAWA and zonal momentum budgets for the monthly extended-winter climatology, and for upward wave pulse events during October, when the winter polar vortex is just starting to get established. We examine the differences between east and west QBO phases, using the JRA-55, and a 100-yr run of the WACCM. In the following subsections we describe the datasets, indices used to define and categorize the events, how statistical significance is determined, and the FAWA diagnostics.

\section{a. JRA-55}

The JRA-55 (Kobayashi et al. 2015) is used for the period 1958-2015 (58 years). This relatively long historical stratospheric reanalysis allows the establishment of a meaningful database for analysis of upward-propagating wave events during fall seasons with east or west QBO phases. The global analyses of daily averaged meteorological variables are used with a spatial resolution of $1.25^{\circ}$ longitude $\times 1.25^{\circ}$ latitude, and 37 isobaric surfaces from 1000 to $1 \mathrm{hPa}$, with 17 levels in the stratosphere (250-1 hPa). In addition, we use daily averaged temperature time tendencies from shortwave (solar) and longwave radiation, provided in the JRA-55 dataset, to directly evaluate the diabatic term in the wave activity budget.

\section{b. The WACCM simulation}

A complementary analysis is provided to compare the key findings in the reanalysis with a free-running WACCM simulation with the setup as described below.

The model simulation is run with NCAR's Community Earth System Model (CESM) version 1.0.2, consisting of atmosphere (WACCM), ocean (POP), land (CLM), and sea ice (CICE) components, based on the Community Climate
System Model (CCSM4; Gent et al. 2011). The atmospheric component used for our experiments is the WACCM version 4 (Marsh et al. 2013) which has a horizontal resolution of $1.9^{\circ} \times 2.5^{\circ}$ (latitude-longitude), 66 levels up to about $140 \mathrm{~km}$, and interactive chemistry [Model for Ozone and Related Tracers (MOZART) version 3]. The chemistry module includes a total of 59 species, such as $\mathrm{O}_{x}, \mathrm{NO}_{x}, \mathrm{HO}_{x}$, $\mathrm{ClO}_{x}, \mathrm{BrO}_{x}$, and $\mathrm{CH}_{4}$, and 217 gas-phase chemical reactions (Marsh et al. 2013). The model has a nudged QBO. The nudging is done by relaxation of the tropical zonal winds between $22^{\circ} \mathrm{S}$ and $22^{\circ} \mathrm{N}$, from 86 to $4 \mathrm{hPa}$ toward an averaged QBO cycle including a relaxation zone to the north and south. The QBO nudging is based on two idealized east QBO and west QBO phases based on observational (rocketsonde) data; see further details in Matthes et al. (2010). Having a QBO in the model is important for a realistic representation of the interaction between the tropical and extratropical region. The solar cycle is prescribed as spectrally resolved daily variations following Lean et al. (2005).

We use a freely running 100-yr simulation (1955-2054) with greenhouse gases (GHGs) and ozone-depleting substances (ODSs) fixed at 1960s concentration levels (pre-ozone hole), with interactive ocean and sea ice components (as in the 3Dozone simulation in Silverman et al. 2018).

\section{c. Defining upward-propagating wave events, during east/west $Q B O$}

Planetary waves exert a major influence on the stratospheric polar vortex, especially during mid- and late winter when the waves are largest, but also earlier on in fall. While the daily climatology of stratospheric waves shows a clear and quite smooth seasonal cycle, with upward wave propagation starting in fall, peaking in midwinter, and ending in spring, the actual wave activity during a given season is quite episodic, with upward wave pulses sometimes lasting only a few days (Plumb 1989; Harnik 2009; Lubis et al. 2016). To examine the effects of these waves, we identify individual wave events based on instances when the meridional eddy heat flux peaks in the midlatitudes in the lower stratosphere. Strictly speaking, the upward EP flux represents the propagation of wave activity into the stratosphere, but it is common to use the meridional heat flux instead (e.g., DunnSigouin and Shaw 2015), since it is proportional to the phase tilt of the waves in the longitude-height plane, and their amplitude, and thus indicates the direction of vertical wave propagation and the magnitude of the waves (e.g., Andrews et al. 1987).

The upward wave events are chosen based on the daily $100 \mathrm{hPa}$ zonal-mean meridional heat flux $\left(\overline{v^{\prime} T^{\prime}}\right)$, averaged between $45^{\circ}$ and $85^{\circ} \mathrm{N}$, weighted by the cosine of latitude, after applying a 5-day running-mean smoothing. The $100-\mathrm{hPa}$ level was chosen since this is a measure of the upwardpropagating wave forcing from the troposphere to the stratosphere (Polvani and Waugh 2004; Charlton and Polvani 2007). Using the $50-\mathrm{hPa}$ level did not qualitatively change our results. Candidate event days were selected for which this heat flux index exceeds the 70th percentile of the daily heat flux during a specific month. Then consecutive days are clustered into a 
single event, requiring more than 5 days separation between individual events. The central day of each event is defined as the day with the largest meridionally averaged $\overline{v^{\prime} T^{\prime}}$ value.

The events were then classified according to the phase of the QBO, which is defined as easterly or westerly using the zonal-mean zonal wind at $50-30 \mathrm{hPa}$, between $2.8^{\circ} \mathrm{S}$ and $2.8^{\circ} \mathrm{N}\left(u_{\mathrm{QBO}}\right)$. As in Silverman et al. (2018), we define the easterly and westerly QBO phase when $u_{\mathrm{QBO}}<-2.5 \mathrm{~m} \mathrm{~s}^{-1}$ and $u_{\mathrm{QBO}}<5 \mathrm{~ms}^{-1}$, respectively. We define a single extended-winter value for each winter, based on the value of winds during October of each winter.

\section{d. Composites, and statistical significance}

Unless specifically noted, all fields shown are anomalies from a daily climatology, calculated from smoothed fields using a 5-day running mean. Two methods are used to calculate the statistical significance of the results presented:

- A Monte Carlo approach is used to determine the statistical significance of composite-mean anomalies. For this, 1000 random samples are created, with each sample having the same number of events or months as in the composite mean. The $95 \%$ confidence levels are then calculated by finding the upper and lower 2.5th percentiles of the produced distribution (see Reichler et al. 2012).

- For the mean difference between two groups (i.e., east/west QBO), statistical significance was calculated using the twosided Student's $t$ test for the composite difference. The figures indicate differences which are statistically significant with a $95 \%$ confidence level.

\section{e. Finite-amplitude wave activity}

The FAWA theory, developed by Nakamura and Solomon (2010), defines a wave activity quantity, as a measure of the change in the amount of quasigeostrophic (QG) PV within the polar cap, bounded by a zonally symmetric PV contour, due to its distortion and nonconservative changes. For each material PV contour we can define an equivalent latitude, $\phi_{e}$, as the latitude at which the surface would be if the PV field were to be rearranged conservatively into a monotonically varying zonal mean state. By construction, the area enclosed by the undulated PV contour is equal to the area enclosed by its equivalent latitude $\phi_{e}(\phi, z, t)$ (which is a function of latitude $\phi$, height $z$ and time $t$ ). Then FAWA is defined as the amount of PV which leaves the polar cap enclosed by $\phi_{e}$, due to the undulation of the PV contour. This is illustrated in Fig. 1 (adapted from Lubis et al. 2018b). We consider a PV contour with a wavy structure, centered around its zonal-mean-state PV value of $Q\left(\phi_{e}\right)$ (bold contour in Fig. 1). The corresponding equivalent latitude $\phi_{e}$ (Butchart and Remsberg 1986; Allen and Nakamura 2003) is

$$
\phi_{q}=\sin ^{-1}\left(\frac{A}{2 \pi a^{2}}-1\right)
$$

where $a$ is the radius of Earth and $A$ is the area enclosed by the latitude circle $\phi_{e}$. The finite-amplitude wave activity is then defined as
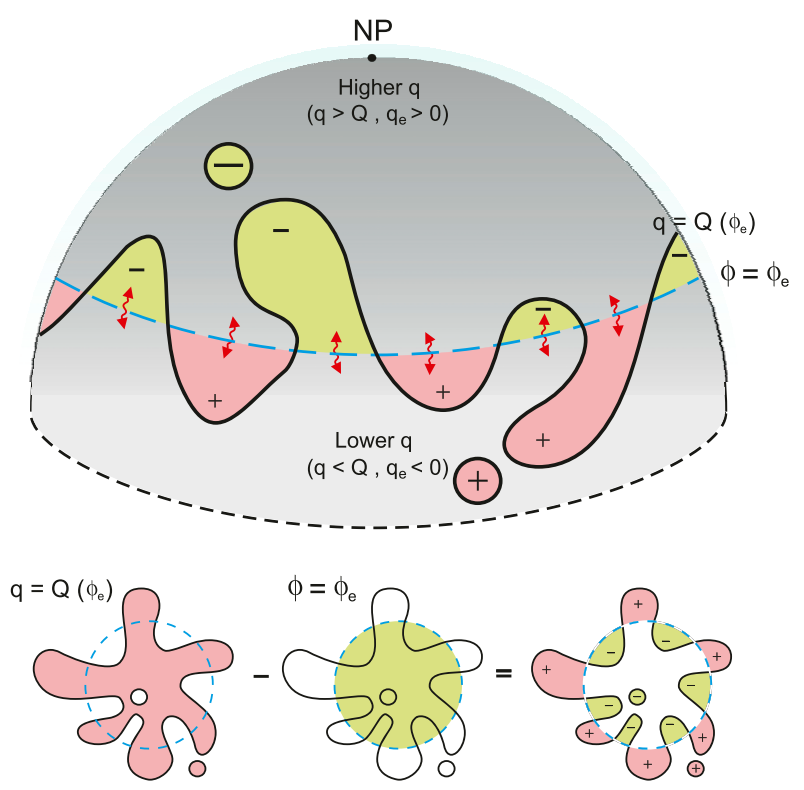

FIG. 1. Schematic of finite-amplitude wave activity on the sphere. Wave activity is defined as the difference in the surface integrals of instantaneous PV from zonal symmetry (over the red regions and below the green regions). The equivalent latitude $\phi_{e}$ is defined such that the red and green areas are equal. The dashed contour is the latitude line $\phi=\phi_{e}$ and the solid contour line of $q=$ $Q\left(\phi_{e}\right)$. The red arrows indicates the eddy fluxes $\left(\overline{v^{\prime} q^{\prime}}\right)$ producing the meridional areal displacement in the $Q$ contour. Adapted from Lubis et al. (2018b).

$$
A^{*}(\phi, z, t)=\frac{1}{2 \pi a \cos \phi_{e}}\left(\iint_{q>=Q\left(\phi_{e}\right)} q d S-\iint_{\phi>=\phi_{e}} q d S\right),
$$

where $d S=a^{2} \cos \phi d \lambda d \phi$ is an area element. From an examination of Fig. 1, this quantity equals the area integral of PV over the red areas, minus the area integrated PV over the green areas. The red and green areas are equal:

$$
\iint_{e>=Q\left(\phi_{e}\right)} d S=\iint_{\phi>=\phi_{e}} d S
$$

Since PV increases monotonically toward the north pole, the PV in the red area is larger than the $\mathrm{PV}$ in the green area, and $A^{*}$, by construction, is positive definite. It is also well defined for highly distorted PV contours, making it applicable also for finiteamplitude eddies.

Two things make FAWA a useful diagnostic of the different contributors to changes in zonal momentum (and the midlatitude polar vortex strength). First, Kelvin's circulation theorem implies that the distortion of a material PV contour will reduce the zonal-mean zonal momentum at $\phi_{e}(\phi, z, t)$ (Solomon and Nakamura 2012), but this reduction will be reversed once the contour is straightened back to its zonal shape, as long as no material PV is lost or gained in the process (i.e., the area enclosed poleward of the PV contour does not change). Thus, an increase in $A^{*}$ occurs at the expense of the zonal-mean zonal flow $\bar{u}$. 
Second, a change in the amount of PV enclosed by $\phi_{e}$ is directly related to the meridional flux of PV through the $\phi_{e}$ latitude circle $\left(\overline{v^{\prime} q^{\prime}} \cos \phi\right)$, which is one of the terms in the transformed Eulerian mean (TEM) formulation of the zonal momentum equation:

$$
\frac{\partial(\bar{u} \cos \phi)}{\partial t}=\overline{v^{\prime} q^{\prime}} \cos \phi+f \bar{v}^{*} \cos \phi+X \cos \phi,
$$

where $\bar{v}^{*}$ is the TEM mean meridional velocity, and $X$ represents nonconservative zonal momentum forcing, which we calculate as a residual.

More explicitly, from the definition of FAWA, its areaweighted change at a given latitude and height results from a poleward flux of PV through the latitude circle, as well as from irreversible dissipation of $\mathrm{PV}$ across the PV contour, and from changes in the PV due to diabatic processes as follows (Nakamura and Zhu 2010):

$$
\frac{\partial A^{*} \cos \phi}{\partial t}=\overline{v^{\prime} q^{\prime}} \cos \phi-K_{\text {eff }} \frac{1}{a} \frac{\partial \bar{q}}{\partial \phi}+\Delta \Sigma \cos \phi .
$$

The irreversible mixing of PV across the distorted material PV contour is expressed as an effective diffusivity with coefficient $K_{\text {eff }}$ times the Eulerian mean PV gradient. In our analysis we calculate this term as a residual of Eq. (5), as was done in previous studies (e.g., Burrows et al. 2017; Lubis et al. 2018b).

The diabatic sources and sinks $\Delta \Sigma$ are calculated explicitly from the diabatic heating rate $J$ as follows:

$$
\Delta \Sigma(\phi, z, t)=\frac{1}{2 \pi a \cos \phi_{e}}\left(\iint_{q>=Q\left(\phi_{e}\right)} \vartheta d S-\iint_{\phi>=\phi_{e}} \vartheta d S\right),
$$

where $\vartheta$ is

$$
\vartheta=\frac{f}{\rho} \frac{\partial}{\partial z}\left(\frac{\rho J}{\bar{\theta}_{z}}\right),
$$

$\rho$ is the density of air, and $\bar{\theta}_{z}$ is the vertical gradient of the zonalmean potential temperature.

In what follows we will use this framework to quantify the processes leading to an irreversible deceleration of the polar vortex by upward pulses of wave activity during fall, and comparing them between east and west QBO.

The irreversible, nonconservative terms are the zonal-mean zonal momentum damping [the $\bar{X}$ term in Eq. (4)], the diabatic FAWA source/sink $[\Delta \Sigma$ term in Eq. (5)] and the diffusive FAWA sink $\left[K_{\text {eff }}\right.$ term in Eq. (5)]. The effect of these irreversible processes can then be examined. For example, by rearranging Eq. (5), we can view the meridional flux of potential vorticity trough a latitude circle as comprising reversible and irreversible parts. The change in FAWA yields a reversible meridional PV flux, meaning, that when the distorted PV contour will return to its circular waveless shape ( $A^{*}$ will decrease), the PV flux will reverse direction. The irreversible part is composed of effective diffusion of PV and diabatic PV sources.

To examine the effect of these irreversible processes on the polar vortex winds, we plug the expression for PV flux from
Eq. (5) into the TEM Eq. (4). This yields the FAWA version of the TEM equation [Nakamura and Zhu 2010; also Eq. (7) in Lubis et al. 2018a]:

$$
\begin{aligned}
\frac{\partial \bar{u} \cos \phi}{\partial t}-f \bar{v}^{*} \cos \phi= & -\frac{\partial A^{*} \cos \phi}{\partial t}-\frac{K_{\text {eff }}}{a} \frac{\partial Q}{\partial \phi_{e}} \cos \phi \\
& +\Delta \Sigma \cos \phi+\bar{X} \cos \phi,
\end{aligned}
$$

where $Q$ is the Lagrangian-mean QGPV with respect to equivalent latitude [bold contour in Fig. 1, see also Eq. (2)].

The acceleration of the mean flow is driven by the wave transience (first term on the rhs), which is reversible, and by a few irreversible processes - mixing of PV (second term on the rhs), diabatic processes (third term on the rhs), and nonresolved zonal wind forcing processes (fourth term on the rhs). To keep the flow in thermal wind balance, changes in the zonalmean zonal wind are partially offset by the Coriolis torque of the residual overturning circulation (second term on the lhs). Charney and Drazin (1961) noted that statistically steady waves (i.e., with constant FAWA), in the absence of diabatic sources and sinks and no friction will not affect the zonal mean flow-the nonacceleration theorem.

Considering an episodic upward-propagating wave entering the midlatitude stratosphere from below, if we take the time integral of Eq. (8) over the full stratospheric wave life cycle, so that the time integral of $A^{*}$ vanishes, the total change of the zonal-mean zonal wind depends on the nonconservative processes and on the offsetting of the acceleration/deceleration by the mean meridional circulation. In this sense the nonacceleration theorem, which strictly applies to steady conservative waves, can be applied to transient waves. Conservative adiabatic waves will have no net effect on the mean flow when integrated over their life cycle (though see caveat discussed below). On the other hand, when the waves are nonconservative (e.g., when they break and their PV is diffusively mixed, or if they are thermally damped), they will irreversibly change the mean flow over their life cycle.

Another way to examine the effects of the waves and the nonconservative processes on the zonal-mean zonal wind is in terms of the reference state, $q_{\text {ref }}$-the PV distribution that would result if the wavy PV contours were instantaneously zonalized (Nakamura and Zhu 2010; Nakamura and Huang 2018). From $q_{\text {ref }}$ we can then obtain the reference zonal-mean zonal wind $u_{\mathrm{ref}}$ by inverting the relation between meridional gradient of mean PV and the zonal-mean zonal wind:

$$
\begin{gathered}
{\left[\frac{1}{f \cos \phi}\left(\frac{\partial^{2}}{\partial \phi^{2}}+\frac{\sin \phi}{\cos ^{2} \phi}\right)+\frac{1}{\rho} \frac{\partial}{\partial z}\left(\rho \epsilon \frac{\partial}{\partial z}\right)\right]} \\
\times\left(\frac{u_{\mathrm{ref}} \cos \phi}{f}\right)=-a \frac{\partial}{\cos \phi \partial \phi}\left(q_{\mathrm{ref}}\right),
\end{gathered}
$$

where $\epsilon=\left(R_{G} \cos ^{2} \phi \bar{\theta}_{z}\right)^{-1}\left(f^{2} a^{2} H\right)$, with $R_{G}$ and $H$ being the gas constant and density scale height, respectively. Equation (9) is inverted to recover $u_{\text {ref }}$, using the no-slip boundary condition as described in Nakamura and Zhu (2010). This was shown to be suitable for the large-scale stratospheric circulation by Lubis et al. (2018b,a). The obtained instantaneous $u_{\text {ref }}$ represents the eddy-free zonal-mean zonal wind field in the absence 
of the effects of the waves on the Eulerian mean PV field. It follows that $u_{\text {ref }}$ can change only due to nonadiabatic sources acting either on the mean flow or on the waves. The conservative effect of the waves on the mean flow can be evaluated as the departure of the observed zonal-mean zonal wind from the defined reference state $\left(u_{\text {ref }}\right)$ (Nakamura and Zhu 2010; Lubis et al. 2018b):

$$
\Delta u \equiv \bar{u}-u_{\text {ref }}
$$

From the above arguments, it follows that $\Delta \bar{u}$ represents the reversible effects of the waves on the mean flow. There is one possible caveat to the association of reversibility to acceleration by conservative processes, that has to do with the overturning circulation. The nonacceleration theorem holds because the conditions for nonacceleration also imply a zero mean meridional circulation (Charney and Drazin 1961). In a temporally varying life cycle setting, where an overturning circulation typically develops in response to the wave entering the domain, it is not clear that the overturning circulation will completely reverse during the stage when the wave leaves the domain, especially since it closes nonlocally, meaning it could be damped outside the stratosphere. Understanding the reversibility of the overturning circulation during stratospheric wave life cycles needs further study. As we will see, however, the contribution of conservative processes to the deceleration of the main polar vortex is quite reversible during October wave life cycles.

Since the FAWA formulation is inherently based on the on the QG approximation it is only applicable poleward of $20^{\circ}$ latitude, and it breaks down in the equatorial region (cf. Solomon and Nakamura 2012; Huang and Nakamura 2017). We note that the quantitative difference between FAWA and its small-amplitude version have been found to be much larger than the differences due to using QG PV compared to primitive equations with Ertel PV in stratospheric studies (Lubis et al. 2018b).

\section{Results}

In what follows we will use the FAWA formulation to examine the climatological monthly mean effects of planetary waves and irreversible processes on the midlatitude polar vortex, and the effect of the QBO phase on these quantities. The life cycles of individual wave pulses during fall will then be analyzed to study their cumulative effect on the stratospheric circulation. Following the findings of Silverman et al. (2018), we expect to see more reversible life cycles during west QBO, indicating the waves are more linear, so that they decelerate the mean flow while they propagate upward into the stratosphere, and accelerate the high-latitude vortex when they deflect toward the subtropics where they decay. Contrarily, when the waves are more nonlinear and they break in the polar vortex region, as during east $\mathrm{QBO}$, the nonconservative terms are expected to be more dominant.

\section{a. The seasonal evolution of finite-amplitude wave activity during opposing $Q B O$ phases}

Figure 2 shows monthly mean fields (not anomalies) from October to February in the Northern Hemisphere, for west
QBO climatology (green contours) and east minus west QBO differences (red-blue shading), using the JRA-55 dataset (1958-2015). The climatology is calculated for each phase of the QBO separately, with the phase being defined based on each year's October zonal-mean zonal wind. Looking first at the west QBO climatology, we see a strengthening of the stratospheric jet from October until December when it reaches its highest intensity (Fig. 2a). The strengthening jet is accompanied by increasing high-latitude wave activity $\left(A^{*}\right)$, which peaks in January (Fig. 2b).

The eddy free reference winds $u_{\text {ref }}$ (Fig. 2c), which show a clear polar vortex structure, increase steadily till January, after which they start weakening. By construction, temporal changes in $u_{\text {ref }}$ are driven by nonconservative processes, acting either on the waves or on the mean flow. The observed increase in $u_{\text {ref }}$ during October-January is consistent, via thermal wind balance, with the seasonal strengthening of the equator-to-pole temperature gradient as solar shortwave heating diminishes over the winter pole. Consistently, $u_{\text {ref }}$ starts weakening in February, as the sun returns to shine over the pole. This radiatively forced acceleration of the vortex is adjusted by an adiabatic wave-driven deceleration ( $\Delta u$, Fig. $2 \mathrm{~d}$ ), which starts being significant in November, and increases throughout winter. As a result, the observed winds are weaker by about one-third compared to the eddy free reference state (cf. Figs. $2 \mathrm{a}$ and $2 \mathrm{c}$ ). We also note the similarity between the spatial structure of $A^{*}$ and $\Delta u$, though with opposite signs, which is expected.

Next we examine the modulation of the seasonal dependence by the QBO, by looking at the east minus west QBO anomalies. We see a significantly weaker jet in the midlatitude upper stratosphere during east QBO (stippled blue regions, Fig. 2a), along with enhanced wave activity at the high-latitude stratosphere during October-January (red regions, mostly poleward of $40^{\circ} \mathrm{N}$, Fig. $2 \mathrm{~b}$ ). At the same time, we see reduced wave activity in the subtropical midstratosphere during October-December, which extends poleward slightly during January-February. We also note that the east-west QBO difference in $\bar{u}$ extends to the troposphere during November, which implies a slightly poleward shifted, and narrower, tropospheric jet during east QBO.

The weaker zonal-mean zonal winds during east QBO in the midlatitude region $\left(10-1 \mathrm{hPa}, 40^{\circ}-60^{\circ} \mathrm{N}\right)$ are driven mostly by enhanced nonconservative wave damping, as indicated by the negative differences in $u_{\text {ref }}$ (Fig. 2c, blue shading), during October-January, which increase in amplitude as winter progresses. During December, however, a significantly positive $u_{\text {ref }}$ anomaly is found at high latitudes. This is consistent with stronger longwave radiation being emitted from the polar night region, which by December is warmer during the east QBO phase. This can explain why the east QBO signal in $\bar{u}$ during December is significant only up to $60^{\circ} \mathrm{N}$. Alongside the weaker high latitude $u_{\text {ref }}$ during east QBO, we see a deep region of stronger $u_{\text {ref }}$ developing between $20^{\circ}$ and $40^{\circ} \mathrm{N}$. This is consistent with previous studies which showed a significant reduction in eddy mixing in the subtropics during east, compared to west QBO (e.g., Shuckburgh et al. 2001). 

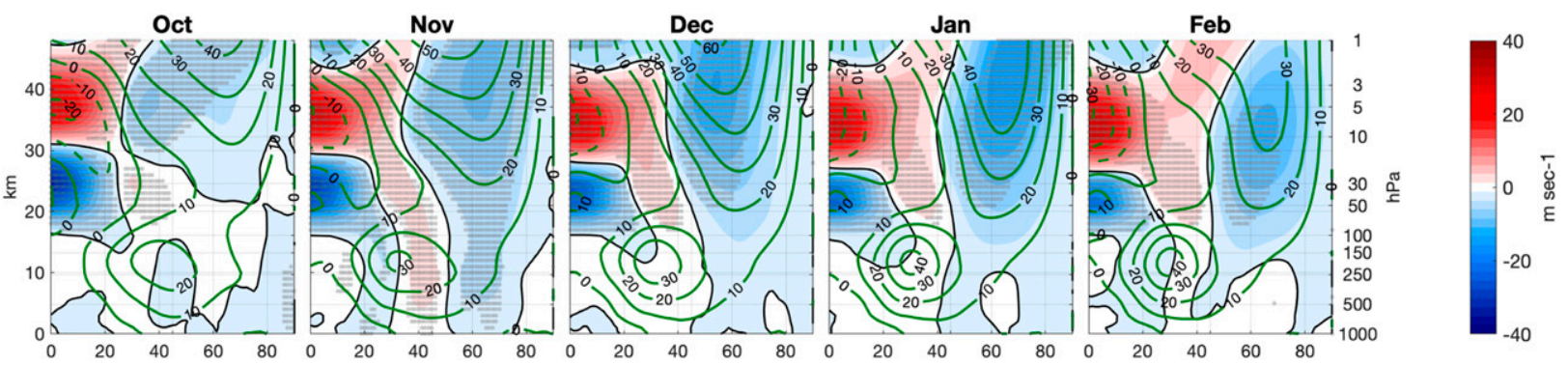

(a) $\bar{u}, \mathrm{E}-\mathrm{W} \mathrm{QBO}$
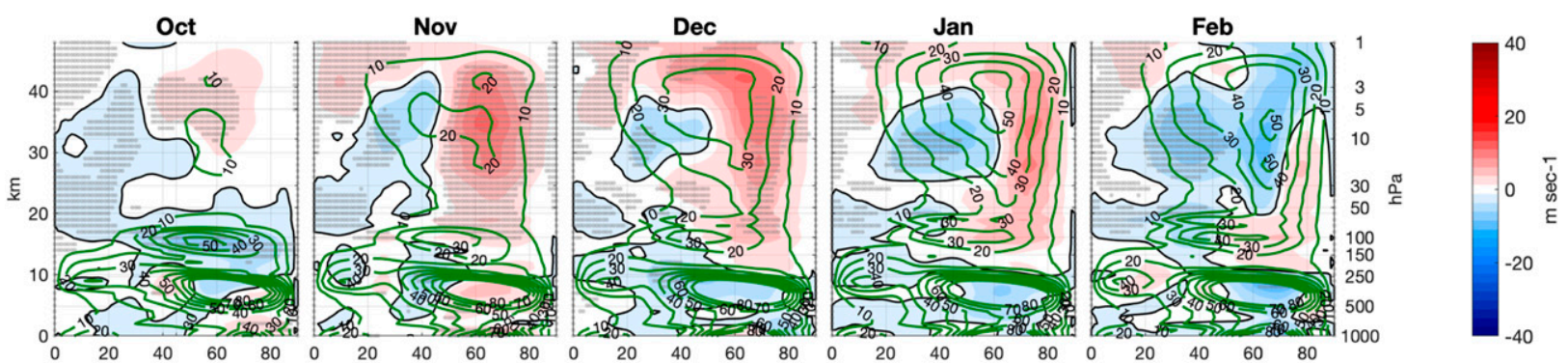

(b) $A^{*}, \mathrm{E}-\mathrm{W} \mathrm{QBO}$
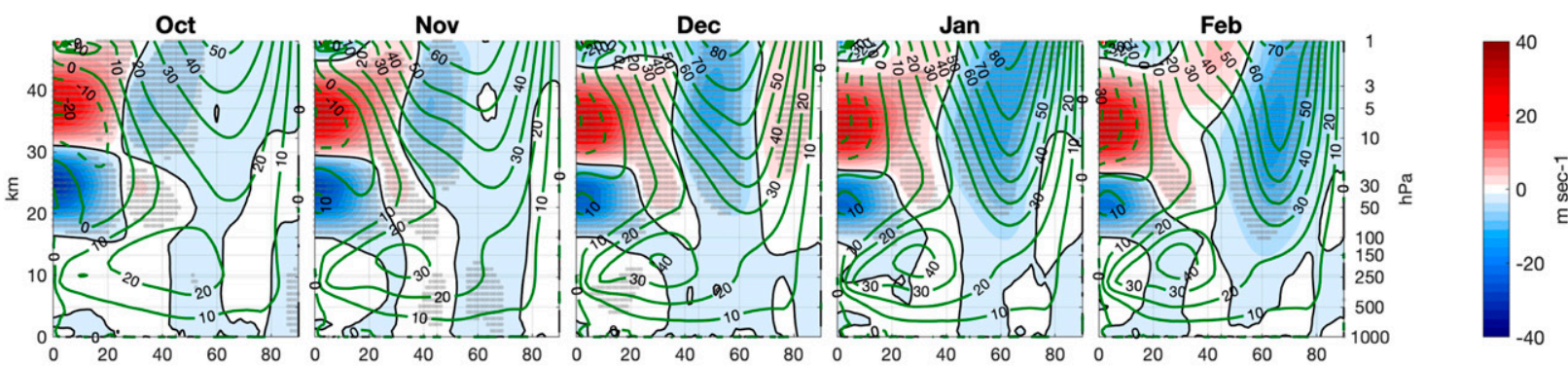

(c) $u_{r e f}, \mathrm{E}-\mathrm{W} \mathrm{QBO}$
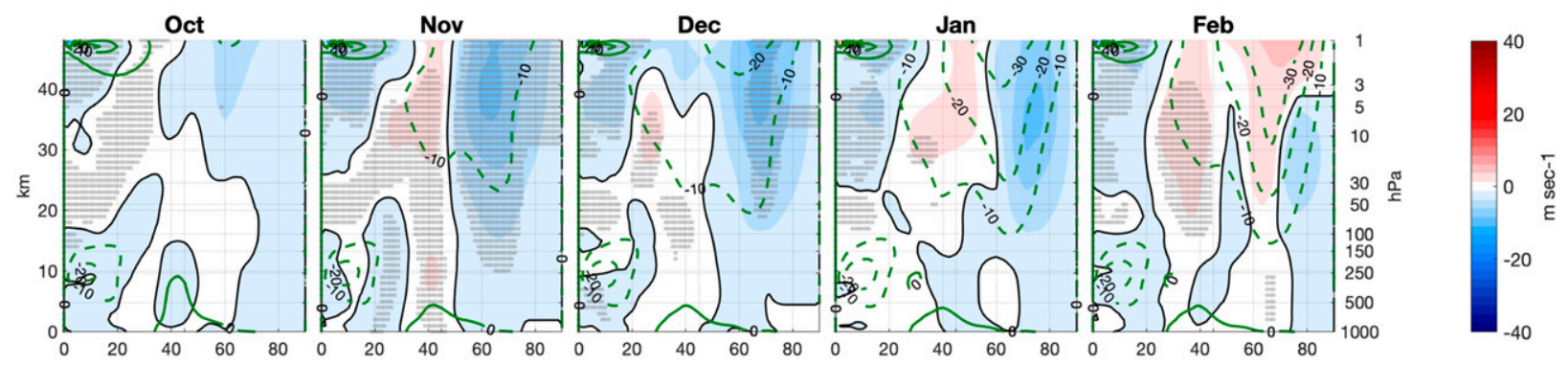

(d) $\Delta u, \mathrm{E}-\mathrm{W} \mathrm{QBO}$

FIG. 2. Monthly climatology of the east-west QBO difference (colors) and west QBO climatology (green contours) of the (a) zonalmean zonal wind $\left(\bar{u}, \mathrm{~m} \mathrm{~s}^{-1}\right),(\mathrm{b}) A^{*}\left(\mathrm{~m} \mathrm{~s}^{-1}\right)$, (c) zonal-mean reference state $\left(u_{\mathrm{ref}}, \mathrm{m} \mathrm{s}^{-1}\right)$, and (d) total zonal-mean zonal wind adjustment to the waves $\Delta u$, October-February, JRA-55, 1958-2015. The dark highlighted contour denotes the zero line of the shaded values. Gray shading indicate statistically significant differences between the east and west QBO phases. 
TABLE 1. The number of positive heat flux events during east and west QBO phases (as defined in section 2c) for October-December inthe JRA-55 and the WACCM model.

\begin{tabular}{lccccc}
\hline & \multicolumn{2}{c}{ JRA-55 } & & \multicolumn{2}{c}{ WACCM } \\
\cline { 2 - 3 } \cline { 6 - 6 } Month & EQBO & WQBO & & EQBO & WQBO \\
\hline October & 27 & 20 & & 55 & 46 \\
November & 37 & 22 & & 52 & 48 \\
December & 33 & 27 & & 52 & 39 \\
\hline
\end{tabular}

The QBO modulation of $\Delta u$ (Fig. 2d) shows a stronger conservative wave-induced deceleration during east QBO, which accounts for the weaker polar vortex at higher latitudes $\left(60^{\circ}-90^{\circ} \mathrm{N}\right)$ during October-November of east QBO years (where $u_{\text {ref }}$ shows no significant signal). At the same time, we see a persistent positive anomaly at lower latitudes, (stippled red shading at $20^{\circ}-40^{\circ} \mathrm{N}$, Figs. $2 \mathrm{c}, \mathrm{d}$ ). This, along with the weaker FAWA in this region during east QBO (Fig. 2b, blue shading), is again indicative of a weaker equatorward refraction of the waves during east QBO, consistent with previous observations and the Holton-Tan mechanism.

To summarize, the extratropical QBO signal, of a weaker vortex during east QBO, starts in the upper stratospheric midlatitudes, and later shifts and extends poleward and downward, with stronger westerly winds developing at lower latitudes $\left(20^{\circ}-40^{\circ} \mathrm{N}\right)$. To better understand these anomalies, we divided the zonal wind changes into those resulting from reversible and irreversible processes; however, once the initial vortex deceleration evolves, it is harder to attribute the observed changes to a specific process since the waves and the mean flow influence each other. During October, however, when the winter polar vortex is starting to get established, and the effect of the waves is still insignificant (e.g., $\Delta u$ during October), we can examine the direct effect of the QBO signal on wave evolution, allowing us to better understand how the midlatitude QBO signal gets initiated. Thus, we next turn to examining the QBO-related differences in the life cycle of upward-propagating wave pulses.

\section{b. The $Q B O$ effect on the reversibility of wave-induced deceleration of the early-season emerging polar vortex}

To examine the effects of upward-propagating waves on the early polar vortex, we identify pulses of upward wave activity as described in section 2c. The total number of upward wave events identified during October, November, and December, for both QBO phases, is shown in Table 1. Our composites comprise more east $\mathrm{QBO}$ wave events [noting there are more east (28) compared to west (23) QBO winters].

Focusing on the wave events found in October in the JRA-55 (central dates shown in Table 2, we examine the time-lagged statistics and composites for various fields around the peak upward heat flux at $100 \mathrm{hPa}$.

Figure $3 \mathrm{a}$ shows the time-lagged composite of anomalous (relative to daily climatology) $45^{\circ}-85^{\circ} \mathrm{N}$-mean, $100-\mathrm{hPa}$, meridional heat flux, alongside the corresponding one standard deviation range for both QBO phases. We see a similarity between the QBO phases in both magnitude and duration. The corresponding
TABLE 2. List of upward-propagating wave events (as defined in section 2c) found in October for east and west QBO phases in the JRA-55.

\begin{tabular}{ll}
\hline \hline EQBO & WQBO \\
\hline 27 Oct 1958 & 31 Oct 1959 \\
15 Oct 1960 & 28 Oct 1961 \\
22 Oct 1965 & 21 Oct 1964 \\
21 Oct 1968 & 20 Oct 1969 \\
27 Oct 1970 & 28 Oct 1971 \\
24 Oct 1972 & 31 Oct 1973 \\
18 Oct 1974 & 24 Oct 1975 \\
24 Oct 1976 & 26 Oct 1978 \\
23 Oct 1977 & 16 Oct 1980 \\
14 Oct 1979 & 29 Oct 1985 \\
31 Oct 1979, & 25 Oct 1987 \\
24 Oct 1981 & 17 Oct 1999 \\
16 Oct 1984 & 31 Oct 2002 \\
27 Oct 1986 & 21 Oct 2004 \\
9 Oct 1989 & 20 Oct 2006 \\
12 Oct 1991 & 31 Oct 2008 \\
5 Oct 1992 & 13 Oct 2010 \\
27 Oct 1996 & 30 Oct 2010 \\
18 Oct 2001 & 29 Oct 2013 \\
5 Oct 2003 & 18 Oct 2015 \\
25 Oct 2003 & \\
22 Oct 2005 & \\
23 Oct 2007 & \\
23 Oct 2012 & \\
13 Oct 2014 & \\
\hline
\end{tabular}

change in the upper stratospheric midlatitude vortex strength, on the other hand, shows a significantly stronger initial deceleration, and a weaker subsequent acceleration during east QBO (Fig. 3b). More explicitly, during east QBO, the winds do not accelerate back to the values they were prior to the wave pulse entering the stratosphere, whereas during west QBO, the vortex essentially recovers to its initial strength. This finding was also found using MERRA-2 (Gelaro et al. 2017; M2T3NPTDT, M2I3NPASM; GMAO 2015a,b), though for a shorter time span (using the events starting in 1980 from Table 2, not shown). We note the clear monthly mean QBO signal-indicated by the fact that the red curves lie above the blue ones for all times (consistent with Fig. 2a). At the same time, the separation between the red and blue curves grows with time, suggesting the individual wave pulses strengthen this preexisting QBO signal.

To separate the wave-induced deceleration from the weak background QBO signal, we examine the change in daily zonal-mean zonal wind anomalies, relative to day -5 , before the wave pulse starts. We examine daily anomalies rather than full fields in order to remove differences related to seasonal changes between wave events occurring during early and late October. Figure 4 shows the latitude-height composite of the zonal-mean zonal wind change from that of day -5 , at different stages of the wave life cycle. By construction, at negative time lags, the wave-induced deceleration is relatively weak, and the QBO signal is evident in the tropics. Around the event center (days -2 to 2) we see wave induced deceleration which extends to high latitudes during both QBO phases. By day 11, however, 


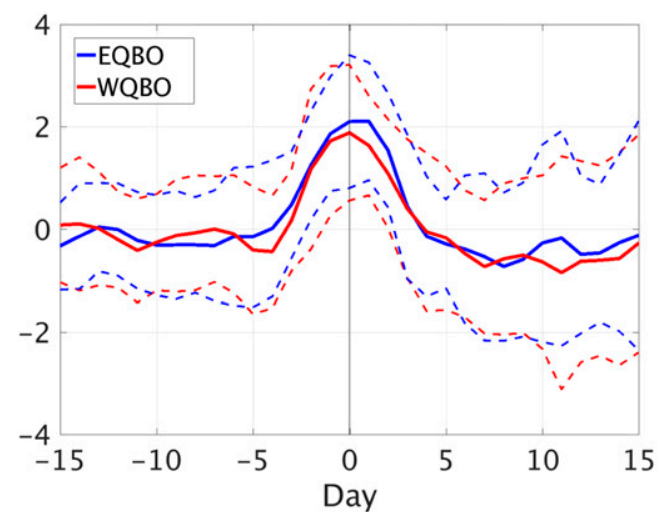

(a) $\overline{V^{\prime} T}\left(\frac{m \cdot K}{s e c}\right), 100 \mathrm{mb}, 85-45 \mathrm{~N}$

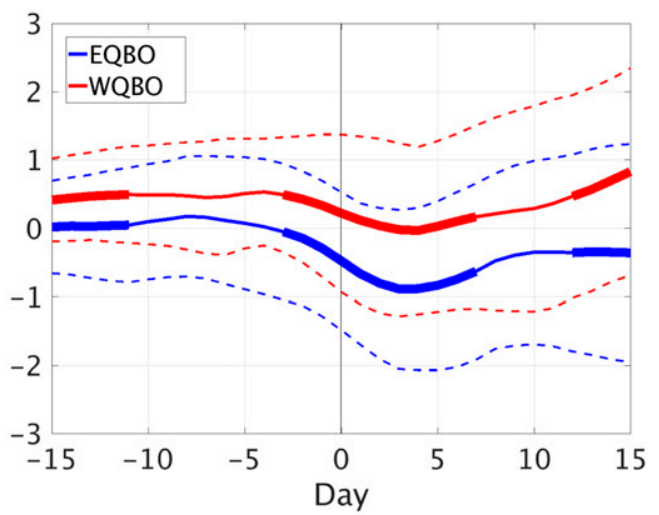

(b) $\bar{U}\left(\frac{m}{s e c}\right), 50-1 \mathrm{mb}, 85-40 \mathrm{~N}$

FIG. 3. Time-lag composites for the upward wave pulse events of (a) $\overline{v^{\prime} T^{\prime}}\left(\mathrm{m} \mathrm{K} \mathrm{s}^{-1}\right)$ averaged over $85^{\circ}-45^{\circ} \mathrm{N}$ at $100 \mathrm{hPa}$, and (b) the zonal-mean zonal wind anomalies $\left(\mathrm{m} \mathrm{s}^{-1}\right.$ ) averaged over $50-1 \mathrm{hPa}, 85^{\circ}-40^{\circ} \mathrm{N}$ (marked by the green rectangle in Fig. 4c), for October events, JRA-55. Dashed lines show \pm 1 standard deviation. Thick lines indicate statistically significant differences between the east and west QBO phases.

the deceleration essentially recovers at high latitudes (poleward of $\left.40^{\circ} \mathrm{N}\right)$ during west QBO. At lower latitudes $\left(20^{\circ}-\right.$ $40^{\circ} \mathrm{N}$ ), on the other hand, significant deceleration develops above $10 \mathrm{hPa}$, which is still significant and growing by days 1115. The resulting east-west QBO anomalies show significantly weaker zonal winds poleward of $40^{\circ} \mathrm{N}$ and significantly stronger zonal winds between $20^{\circ}$ and $40^{\circ} \mathrm{N}$.

A weak tropospheric influence is also notable on the subtropical jet (climatologically located around $40^{\circ} \mathrm{N}$, see Fig. 2a), showing different acceleration/deceleration for east/west QBO (and are partially statistically significant during the later stages of the life cycle). This suggest a tropospheric response to the upward-propagating stratospheric wave events, which is QBO dependent. To understand the tropospheric response more analysis needs to be done which is beyond the scope of this paper. However, we wish to point out the similar pattern of the east-west anomalies found in the midlatitude troposphere in the life cycle composite (Fig. 4c) and the November climatology (Fig. 2a), indicating the possibility that these different tropospheric feedbacks from the wave life cycle accumulate to later on form the November east-west climatology.

To further examine the differences between the waveinduced effects at high- and low-latitude stratosphere, we calculate mass-weighted averages in the two regions marked by the green and orange boxes in Fig. 4c. Figure 5 shows the wave-induced changes, relative to day -5 , of the daily anomalies of $\bar{u}, A^{*}, u_{\text {ref }}$, and $\Delta u$, for east and west QBO phases, in each of the two regions. Looking first at the evolution in the higher-latitude region, during east QBO events, we see a growth in wave activity (black curves) and a concurrent zonal-mean flow deceleration (green curves), which reverse from day 5 , but do not reach zero values (though the anomalies stop being significant beyond days 8-9). During west QBO, on the other hand, the growth in $A^{*}$ is weaker, and the mean flow deceleration is fully reversible $\left(u_{\text {ref }}\right.$ is nearly constant with time, and the zonal-mean wind anomalies are zero by day 14$)$. We note that averaging over a high-latitude box $\left(60^{\circ}-85^{\circ} \mathrm{N}\right)$ shows a reversible deceleration also for the east QBO phase, suggesting the waves get absorbed in the mean flow equatorward of $60^{\circ} \mathrm{N}$, consistent with the observed deceleration at days 11-15 (Fig. 4a).

At lower latitudes, for both QBO phases, we see that $A^{*}$ starts increasing only by day 1 (as opposed to negative time lags at higher latitudes), and it reaches a smaller magnitude compared to higher latitudes (especially during east QBO), before starting to decrease. This is consistent with the waves first propagating up to the stratosphere at higher latitudes, then refracting to lower latitudes. The corresponding mean flow deceleration, however, is different between the two QBO phases, in a way which is somewhat opposite to that at higher latitudes. During east QBO, the deceleration in $\bar{u}$ is driven by conservative processes $(\bar{u}$ and $\Delta u$ are nearly equal, though anomalies are not statistically significant, and both reverse and even seem to overshoot (showing statistically significant difference compared to the west QBO). During west $\mathrm{QBO}$, on the other hand, the deceleration is initially driven by conservative processes, but by day 7 , nonconservative processes increase (evident as negative $u_{\text {ref }}$ anomalies-cyan line), while the $\Delta u$ anomalies remain quite large, resulting in the total deceleration continuing throughout the 15 days shown. Overall, about $30 \%$ of this deceleration is driven by nonconservative processes, while most if it is due to conservative wave driving. As noted before (at the end of section 2e), the fact that the $\Delta u$ anomalies do not reverse as they do at higher latitudes is not fully understood, and needs further research.

To further understand the sources of the differences between the QBO phases in the main vortex region, we calculate the high-latitude box averaged composites of the different terms in the FAWA and zonal-mean zonal momentum budgets [Eqs. (4) and (5)]. Figure 6 presents three different budgets. The top row shows the zonal-mean zonal momentum budget, with the signs of the different terms 

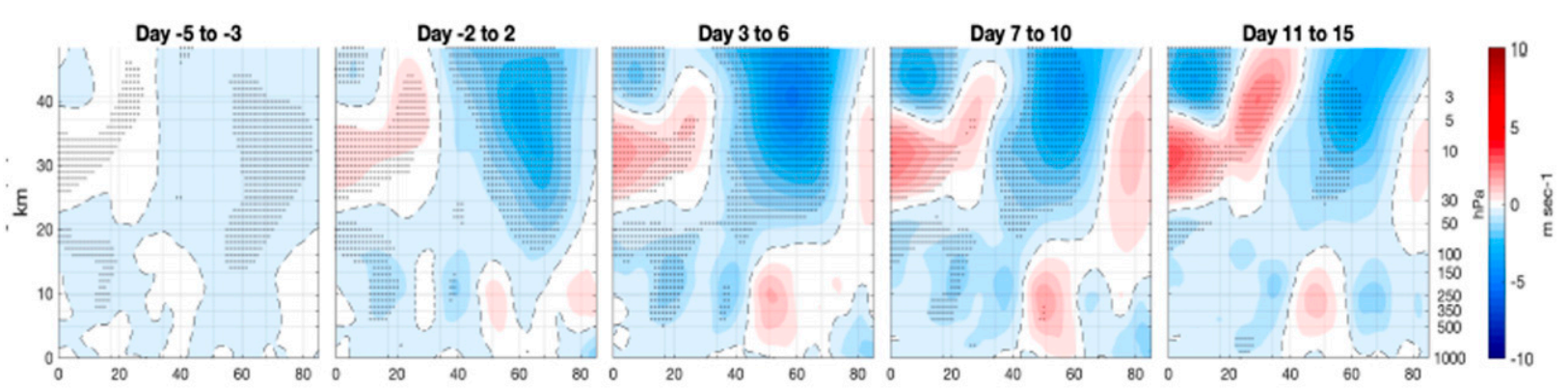

(a) $u_{o b s}, \mathrm{EQBO}$
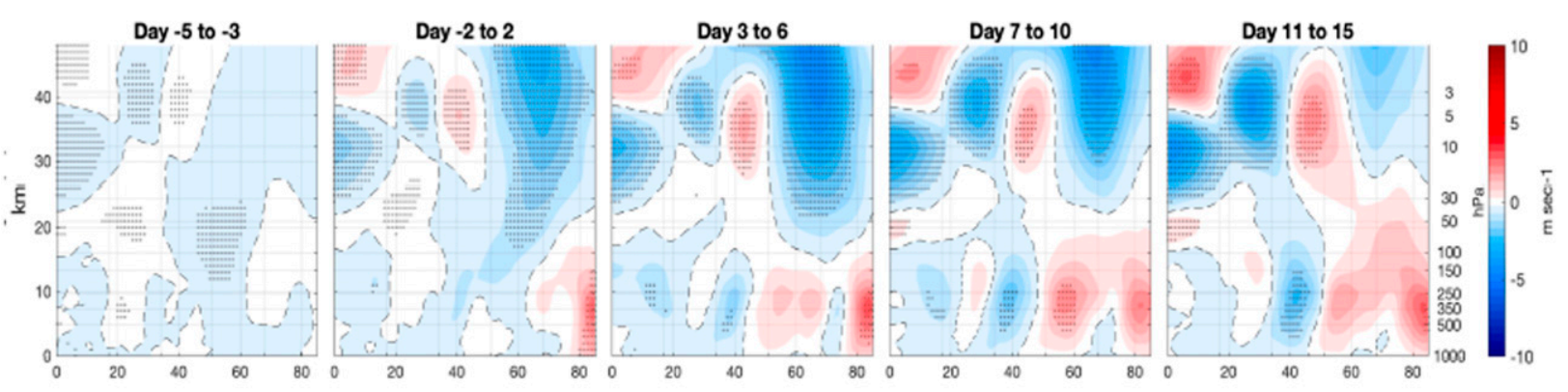

(b) $u_{\text {obs }}$, WQBO
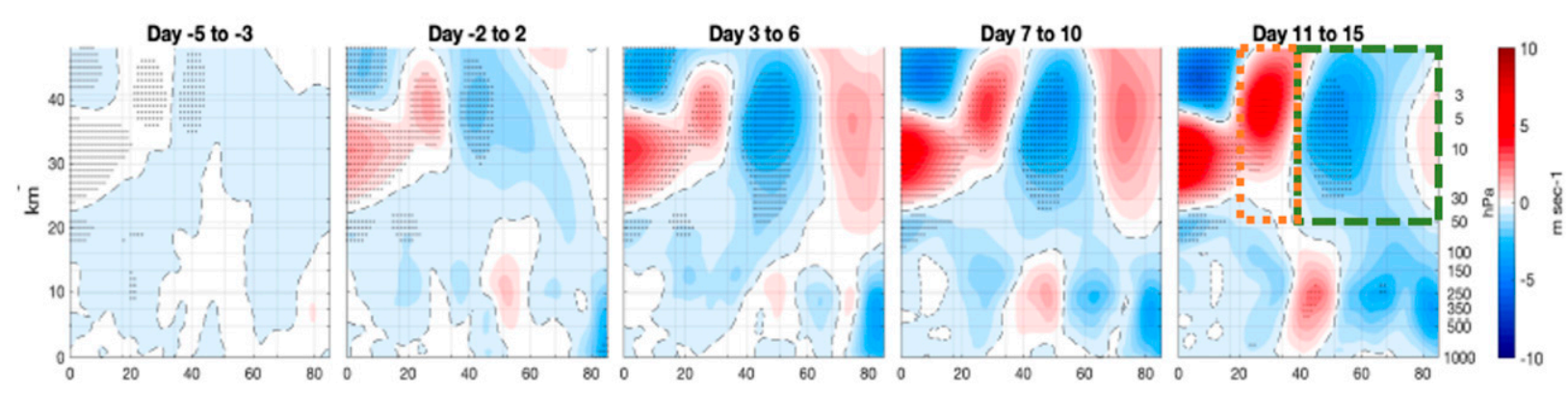

(c) $u_{o b s}, \mathrm{E}-\mathrm{W} \mathrm{QBO}$

FIG. 4. Latitude-height time-lag composites of the zonal-mean zonal wind $\left(\bar{u}, \mathrm{~m} \mathrm{~s}^{-1}\right)$, anomalies from the climatology with respect to anomalies at lag -5 days, for (a) east QBO, (b) west QBO, and (c) east-west QBO, for the positive heat flux events (70th percentile of $\overline{v^{\prime} T^{\prime}}$ at $100 \mathrm{hPa}, 85^{\circ}-45^{\circ} \mathrm{N}$ ) during October, JRA-55. Gray shading indicates statistically significant differences between (a) and (b). The green and orange boxes in (c) show the area of averaging for the composites in Figs. 3 and 5.

calculated so that the area-averaged $\bar{u}$ time tendency (green line) equals the sum of all other terms. We see a statistically significant stronger deceleration during east QBO life cycles. During both QBO phases, the deceleration and subsequent acceleration are partially balanced by the Coriolis torque. The major difference between the life cycle budgets of the two QBO phases is in the contribution of nonconservative wave drag, which is significantly stronger (in absolute value), and negative (decelerating) during east QBO, whereas it is weak and positive (accelerating) during west QBO. Time integral over days -5 to 15 (indicated in parentheses in the legend), shows the mean acceleration over the wave life cycle, and the contribution to it from the different terms. We see a net acceleration of the zonal winds (positive change) during west $\mathrm{QBO}{ }^{2}$ while there is a net deceleration during east QBO.

The second row in Fig. 6 shows the decomposition of the meridional PV flux into its different components, as obtained

\footnotetext{
${ }^{2}$ This "overshooting" of the zonal wind acceleration may be due to the seasonal cycle being stronger during west QBO events (recall that the anomalies are calculated relative to the daily climatology based on all years). This may also explain why the composites are statistically significant beyond 15 days during west QBO; however, understanding this is left for future study.
} 


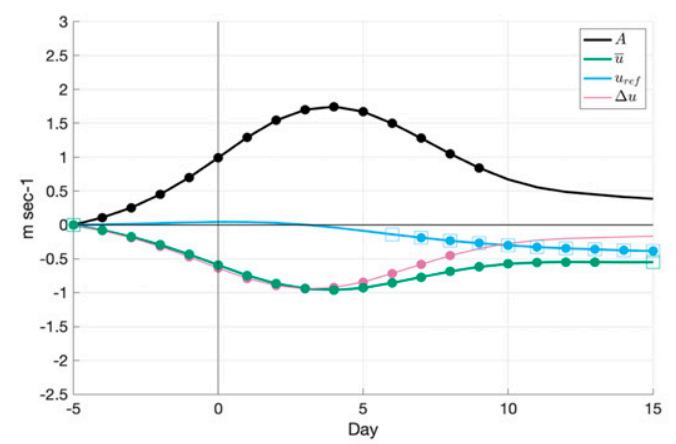

(a) EQBO, $85-40 \mathrm{~N}$

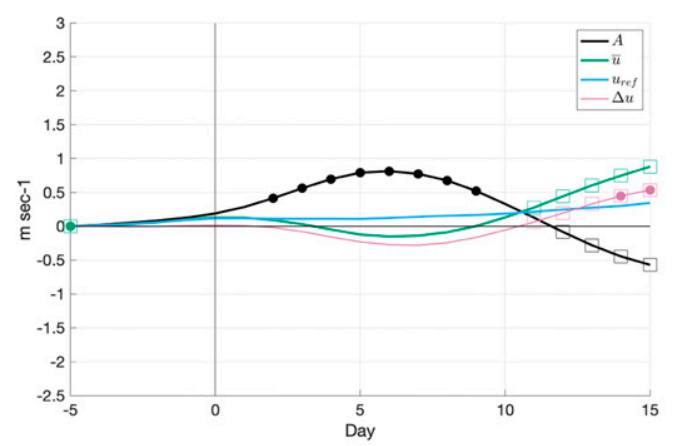

(c) EQBO, 20-40N

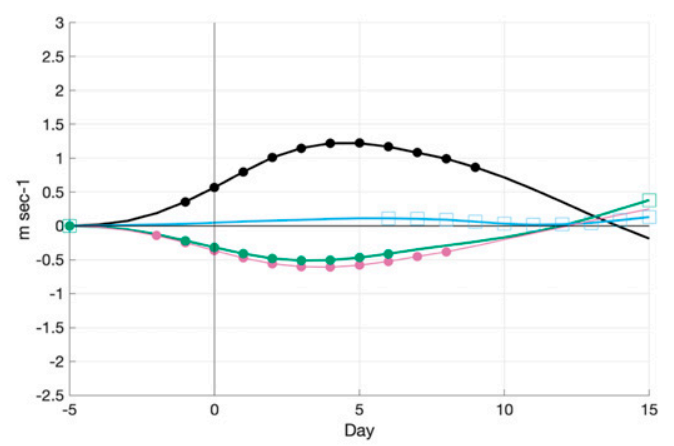

(b) WQBO, $85-40 \mathrm{~N}$

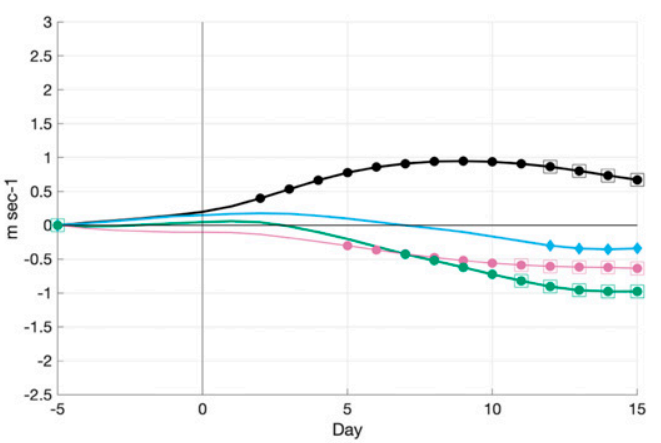

(d) WQBO, 20-40N

FIG. 5. Time-lag composites for the accumulated change (anomalies with respect to lag -5 days) in the zonalmean zonal wind $\bar{u}$ (green), $A^{*}$ (black), $u_{\text {ref }}$ [the change in winds due to nonconservative processes (blue)], and $\Delta u$ [the adiabatic contribution to the wind tendency (pink)], averaged over (top) $85^{\circ}-40^{\circ} \mathrm{N}$ and (bottom) $40^{\circ}-20^{\circ} \mathrm{N}$ at 50-1 hPa for upward wave pulse events during October for (left) east QBO and (right) west QBO, JRA-55. Statistically significant values relative to the climatology are indicated by filled circles. Statistically significant differences between east and west QBO life cycles are marked by empty squares.

from rearranging Eq. (5) to have the PV flux on the left-hand side. Viewed this way, the PV flux (black line) is a sum of all other terms plotted. For both QBO phases, we see that $\overline{v^{\prime} q^{\prime}}$ first decreases (growing waves are associated with a downgradient, negative, PV flux) then increases, and this evolution mostly results from the increase then decrease in FAWA (which is plotted with a minus sign), with some contributions from diffusive PV mixing, and during east QBO, also a small contribution from diabatic processes. Similar to the mean flow dissipation (turquoise line in top row), the mixing term is negative and significantly stronger during east QBO, and is weak and positive during west QBO. The similarity between the eddy and mean flow dissipation terms suggest they result from similar processes.

The combined effect of nonconservative processes is shown in the third row of Fig. 6, where we plot the different terms in Eq. (8), rearranged to have $(\partial / \partial t)\left(\bar{u}+A^{*}\right)$ on the left-hand side, and all the damping and diabatic heating terms are combined into one term representing the overall contribution from nonconservative processes. The quantity $\bar{u}+A^{*}$, which we will refer to as the wave-modified zonal wind, is related to $u_{\text {ref }}$ via Eq. (9), and the following relation which can be derived from the definition of $A^{*}$ (cf. Fig. 1):

$$
\int_{\phi_{e}} \bar{q}_{\mathrm{ref}} \cos \phi_{e} d \phi_{e}=\int_{\phi} \bar{q} \cos \phi_{e} d \phi+A^{*} \cos \phi
$$

From Eq. (8), the changes in the wave-modified zonal wind, once the counterresponse of the Coriolis term is taken into account, are driven by nonconservative processes (acting both on the zonal mean and on the waves). We see that during both QBO phases, as the wave activity increases in the domain, the wave-modified zonal wind initially grows (due to the fact that part of the wave-induced deceleration is offset by the overturning circulation, which is excited to preserve thermal wind balance), and when the wave activity starts decaying, the wavemodified zonal wind decays back. We also see that the change from growth to decay occurs earlier during east $\mathrm{QBO}$, due to a large and significant contribution of nonconservative terms, which are essentially zero during west QBO.

In the absence of nonconservative processes, either on the mean flow or on the waves, a wave packet, which enters the domain from below, will fully leave it, either from its southern boundary, as it is refracted toward the equator, or upward to the mesosphere. As a result, the wave transience contribution to the deceleration by the upward-propagating wave will be fully reversed. Assuming the corresponding overturning 


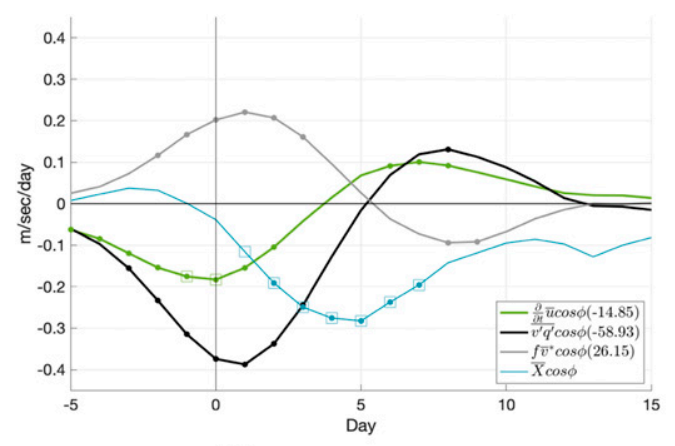

(a) $\frac{\partial U}{\partial t}$ budget, EQBO

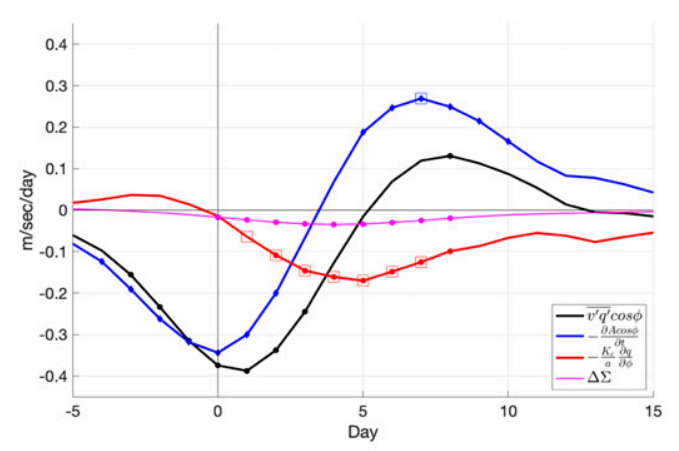

(c) $\overline{v^{\prime} q^{\prime}}$ budget, EQBO

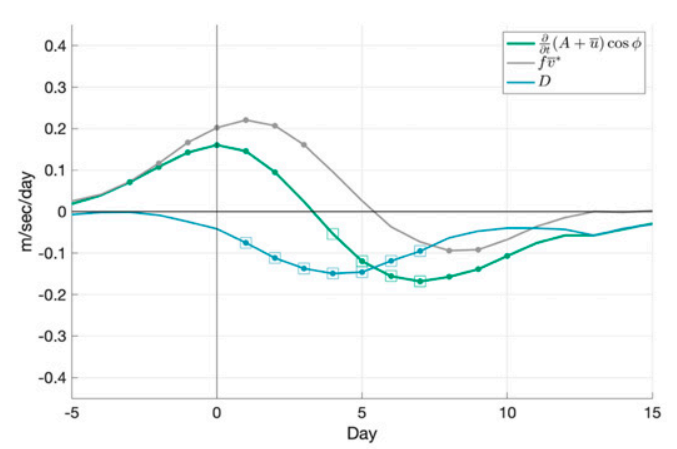

(e) $\frac{d}{d t}(\bar{u}+A)$ budget, EQBO

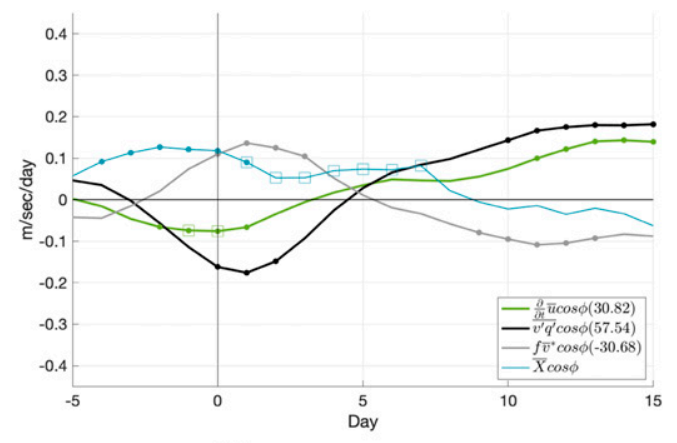

(b) $\frac{\partial U}{\partial t}$ budget, WQBO

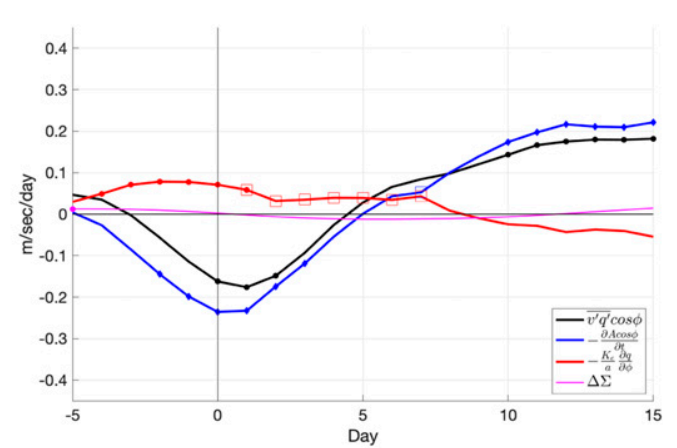

(d) $\overline{v^{\prime} q^{\prime}}$ budget, WQBO

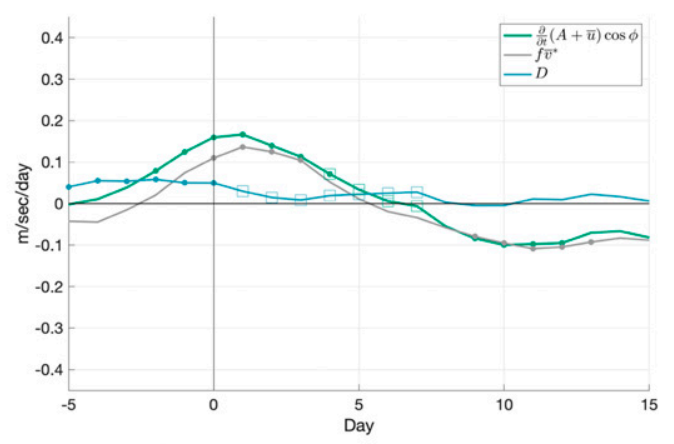

(f) $\frac{d}{d t}(\bar{u}+A)$ budget, WQBO

FIG. 6. Time-lag composites for (a),(b) the zonal momentum budget [m (s day) $\left.{ }^{-1}\right]$, (c),(d) the FAWA budget [m (s day) ${ }^{-1}$ ] showing $\overline{v^{\prime} q^{\prime}} \cos \phi$ (black), $(\partial \bar{u} / \partial t) \cos \phi, f \bar{v}^{*} \cos \phi$ (gray), $-\partial A^{*} \cos \phi / \partial t$ (blue), $-\left(K_{\text {eff }} / a\right)(d \bar{q} / d \phi) \cos \phi$ (red), and $\Delta \Sigma$ (magenta), and (e),(f) the nonacceleration relation $(d / d t)(\bar{u}+A)\left[\mathrm{m} /\left(\mathrm{s} \mathrm{day}^{-1}\right)\right]$ (green), the Coriolis torque $\left.[\mathrm{m} \text { (s day })^{-1}\right]$ (gray), and the nonconservative terms $\left[\bar{X}+\Delta \Sigma-\left(K_{\mathrm{eff}} / a\right)(d \bar{q} / d \phi)\right] \cos \phi\left[\mathrm{m} /(\mathrm{s} \text { day })^{-1}\right]$ (blue). Averaged over 85-40 N, 50-1 hPa, for upward wave pulse events during October for (left) east QBO and (right) west QBO, JRA-55. Statistically significant values relative to the climatology are indicated by filled circles. Statistically significant differences between east/west QBO life cycles are marked by empty squares.

circulation which arises to keep thermal wind balance also fully reverses, the final mean flow will be similar to what it was prior to the wave entering the stratosphere. This is the transient version of the nonacceleration theorem, which, strictly speaking, was derived for statistically steady waves (Charney and Drazin 1961). Figure 6 suggests nonacceleration conditions apply quite well for waves propagating into the stratosphere during Octobers of west QBO years, suggesting the overturning circulation effect is also reversible. An explicit calculation of $f \bar{v}^{*}$ averaged over this box (not shown) also supports this.

These results are consistent with those found by Silverman et al. (2018) (Fig. 12) using the small-amplitude enstrophy budget in WACCM. In that study, we found that during west 

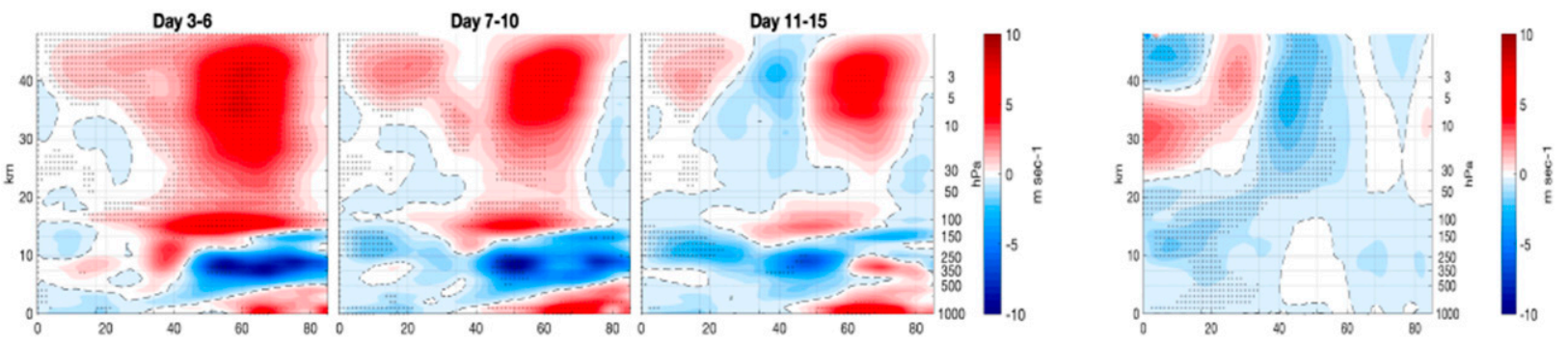

(a) $A^{*}, \mathrm{EQBO}$

(b) $u_{\text {ref }}, \mathrm{EQBO}$
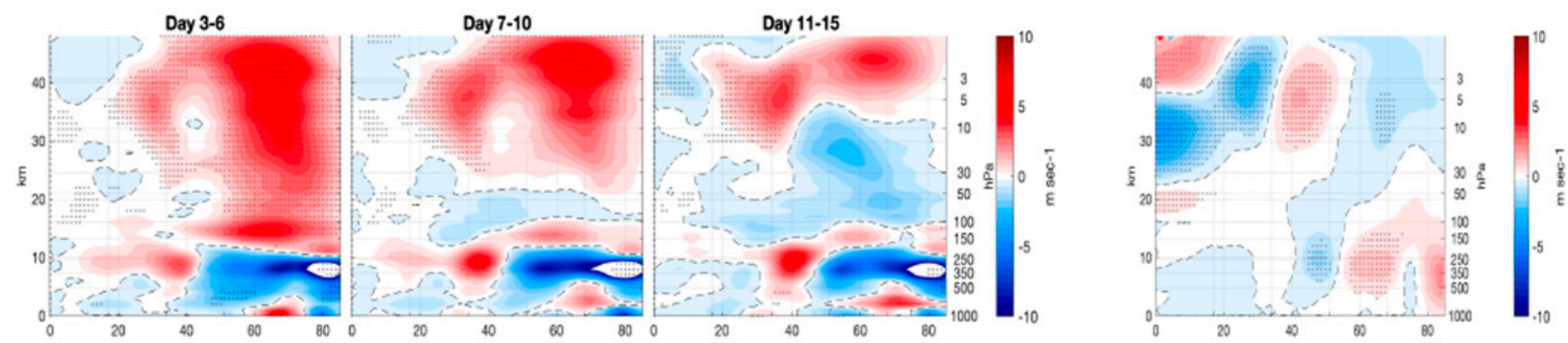

(c) $A^{*}$, WQBO

(d) $u_{r e f}$, WQBO

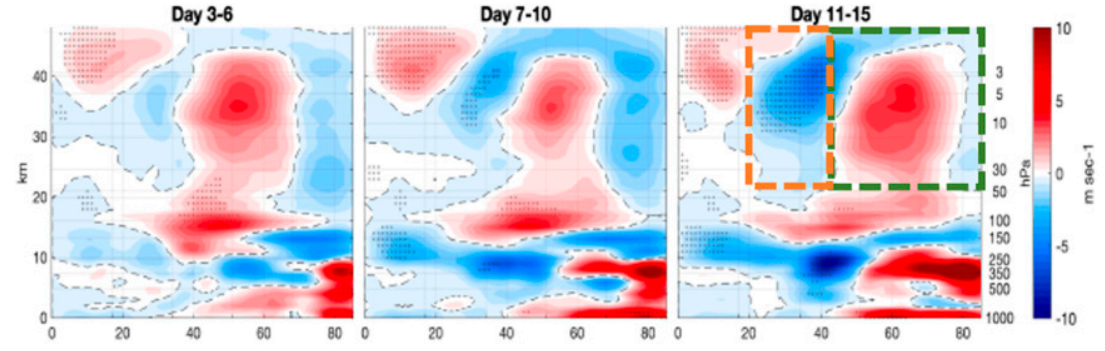

(e) $A^{*}, \mathrm{E}-\mathrm{W}$ QBO

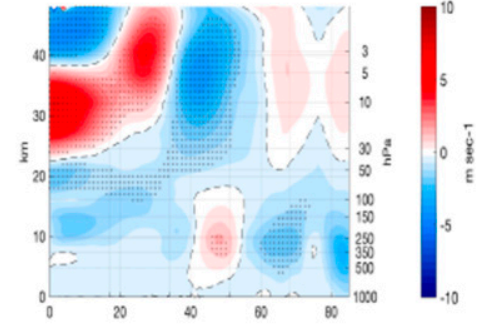

(f) $u_{r e f}, \mathrm{E}-\mathrm{W} \mathrm{QBO}$

FIG. 7. Latitude-height time-lag composites of (a),(c),(e) $A^{*}\left(\mathrm{~m} \mathrm{~s}^{-1}\right)$ (days 3-15) and (b),(d),(f), $u_{\mathrm{ref}}\left(\mathrm{m} \mathrm{s}^{-1}\right)($ days $11-15)$ anomalies from the climatology with respect to anomalies at lag -5 days, showing (top) east QBO, (middle) west QBO, and (bottom) the east-west QBO differences, for positive heat flux events (70th percentile of $\overline{v^{\prime} T^{\prime}}$ at $100 \mathrm{hPa}, 85^{\circ}-45^{\circ} \mathrm{N}$ ) during October, JRA-55. (top),(middle) Gray shading indicates statistically significant anomalies (relative compared to the climatology) and (bottom) east-west QBO differences. The green and orange boxes in (e) show the area of averaging for the composites in Figs. 3 and 5.

QBO, waves refract equatorward before dissipating, whereas during east QBO, the waves dissipate in the high-latitude stratosphere. To explicitly check if this also happens in JRA55, we examine the latitude-height FAWA $\left(A^{*}\right)$ anomaly composites at different stages of the wave life cycle. Figure 7 shows the change in daily FAWA anomalies relative to day -5 of each wave event, during the peak of the deceleration (days 3-6) and at two later stages of the wave life cycle (days 7-10, days 11-15). During both QBO phases, the FAWA anomalies, which at days 3-6 occupy most of the midlatitude stratosphere, shift upward, diminishing from the midlatitude stratosphere by days 11-15 (note the positive anomaly during east QBO is not statistically significant). There is a clear difference, however, in how the FAWA evolves. At days 3-6 we see an equatorward expansion of FAWA which occurs in the upper stratosphere (above $5 \mathrm{hPa}$ ) during east QBO. Later on, as FAWA diminishes, the subtropical peak separates from the high-latitude
FAWA center, and by days $11-15$, only the upper stratospheric subtropical center remains statistically significant. During west QBO, on the other hand, the FAWA center propagates upward and equatorward, so that by days $11-15$, there is significantly more FAWA in the low-latitude box shown in Figs. 5c and 5d. The different locations of the FAWA centers implies different regions where nonconservative wave processes can irreversibly decelerate the mean flow. This is shown in the rightmost column of Fig. 7, where we have plotted the $u_{\text {ref }}$ anomalies during days $11-15$. We note that during east $\mathrm{QBO}$, nonconservative processes are significant only at lower latitudes $\left(35^{\circ}-50^{\circ} \mathrm{N}\right)$ while at higher latitudes, the deceleration is driven by reversible wave processes (a box average of the zonal momentum and FAWA budgets over $60^{\circ}-80^{\circ} \mathrm{N}$ confirms this, not shown). During west QBO, nonconservative deceleration is strong in the low-latitude box, and coincides with the FAWA peak there, suggesting the 
main contributing process is enhanced wave damping. We also note that there are strong and significant positive $u_{\text {ref }}$ anomaly centers which form in the upper stratosphere, between $20^{\circ}$ and $25^{\circ} \mathrm{N}$ during east QBO, and between $40^{\circ}$ and $55^{\circ} \mathrm{N}$ during west QBO. These acceleration regions, which lie right to the north or south of the main $u_{\text {ref }}$ deceleration centers, may be due to the irreversible wave mixing which flattens out the PV gradients, causing the PV gradients to sharpen at the edge of the mixing domain, and thus to strengthening the jet [cf. Eq. (9)].

The above evolution of $A^{*}$ and $u_{\text {ref }}$ are consistent with the findings of Silverman et al. (2018), that wave dissipation occurs more equatorward during west compared to east QBO. There are differences, however, in the evolution of the wave life cycles, especially during the west QBO, between the life cycles presented here and those we analyzed in the WACCM model in Silverman et al. (2018). This will be discussed next.

\section{c. The $Q B O$ effects in WACCM}

The Holton-Tan effect is an important teleconnection phenomenon necessary to properly capture the variability of the stratosphere, and, at certain cases, a subsequent tropospheric response. There is a large variation, however, in the ability of GCMs to simulate this effect (Anstey et al. 2021). In this section we repeat the analysis using the WACCM runs from Silverman et al. (2018) and show there are a few significant differences in the seasonal evolution of the Holton-Tan effect, as well as in the life cycles of upward wave pulses during October, relative to JRA-55. Despite these differences, however, the main findings for JRA-55 hold, namely, that there is more irreversible wave mixing at higher latitudes during east QBO, while during west QBO, nonacceleration conditions hold in the main high-latitude vortex region, with wave-induced deceleration occurring at lower latitudes where the waves dissipate.

Figure 8 shows the monthly mean climatology for west QBO, alongside the east minus west QBO differences, as was shown for JRA-55 in Fig. 2. The main seasonal evolution is similar to JRA-55; namely, there is a stronger deceleration alongside stronger a FAWA, at high latitudes during east QBO. However, the seasonal evolution occurs faster in WACCM, with the peak east-west $\bar{u}$ anomaly occurring in December, compared to January in JRA-55. This is likely because the climatological waves in WACCM are stronger and they peak higher up, starting already in October. This results in an overall weaker vortex (for both QBO phases), and a stronger east-west QBO difference in vortex strength in WACCM. As a result, in WACCM, the $A^{*}$ anomaly changes sign in January, meaning it is weaker during east QBO (due to the much weaker vortex), and correspondingly, the January east-west QBO difference in $\bar{u}$ weakens to near zero. This oscillation of the polar night jet-wave amplitude is similar to the polar night jet oscillation, found by Kuroda and Kodera (2001) to be characteristic of the internal stratospheric wave-mean flow variability. In JRA-55, we see a similar change occurring a month later, in February (negative east-west $A^{*}$ anomaly alongside a weakening in the east-west difference in $\bar{u}$ ).
Another difference is the positive high-latitude east-west anomaly in $u_{\text {ref }}$ during October-November (and in the lower polar stratosphere during December) in WACCM, which in JRA-55 only appears during December. We suspect this has to do with the radiative cooling of the polar region being stronger during east $\mathrm{QBO}$, as a result of the polar region being warmer. We note that the WACCM simulation was run using preindustrial concentrations of greenhouse gasses and ozone depleting substances, which may account for some of the above differences. Overall, however, the wavemean flow dynamics, and the QBO signal, are qualitatively similar during October, allowing us to compare the upwardpropagating wave life cycles, and how they are influenced by the QBO phase.

To examine the early onset of the midlatitude QBO effect, we composite different fields around the peak upward wave pulses entering the stratosphere from below, as was done for JRA-55 (using the definitions described in section 2b). Since many of the results are similar, we only present the main differences from JRA-55. For clarity, we present the results for the JRA-55 domain, even though WACCM extends much higher (up to $0.001 \mathrm{hPa}$ ). Figure 9 shows the anomaly relative to day -5 of $\bar{u}$ anomaly, averaged over days 11-15. This quantity represents the change in the vortex strength, remaining after the upward wave pulse has decelerated and accelerated the vortex over its life cycle. During east QBO, we see a significant midlatitude deceleration, which extends more equatorward for WACCM (Figs. 9a,d During west QBO, however, we see much larger differences (cf. Figs. 9b,e). For both datasets, we see a low-latitude deceleration alongside an acceleration immediately poleward of it (forming a latitudinally oriented deceleration-acceleration dipole). The dipole centers, however, are shaped and located differently between the datasets. While in JRA-55 the easterly (westerly) anomalies peak at around $3-5 \mathrm{hPa}$, between $20^{\circ}$ and $40^{\circ} \mathrm{N}\left(40^{\circ}\right.$ and $\left.60^{\circ} \mathrm{N}\right)$, and both are statistically significant, in WACCM, the easterly (westerly) centers peak around $0.1 \mathrm{hPa}$, between $20^{\circ}$ and $50^{\circ} \mathrm{N}\left(50^{\circ}\right.$ and $\left.90^{\circ} \mathrm{N}\right)$, and essentially only the easterly center is statistically significant (there are small regions of statistically significant westerly anomalies at the top polar corner and also above the domain shown). Correspondingly, the east-west QBO difference in JRA-55 is a downward shifted, latitudinally compressed version of the WACCM pattern (cf. Figs. 9c, f We also see significant differences in the tropospheric response. During east QBO, both datasets show a dipole of westerlies (around $50^{\circ} \mathrm{N}$ ) and easterlies (around $70^{\circ} \mathrm{N}$ ) spanning the troposphere from the surface to $150 \mathrm{hPa}$, which are statistically significant mostly in WACCM. During west QBO, there is a significant easterly anomaly (centered around $40^{\circ} \mathrm{N}$ ) in JRA-55, while in WACCM there is a much weaker and hardly statistically deceleration center more equatorward (around $25^{\circ} \mathrm{N}$ ). Similar to the findings in JRA-55, we note that the tropospheric anomalies are consistent with the monthly mean anomalies seen in November (Figs. 2, 8) suggesting the QBO dependent tropospheric effect of October life cycles, may contribute to the monthly mean anomalies later on.

Consistent with the above differences in the $\bar{u}$ composites, the composited $A^{*}$ anomalies show some qualitative differences 

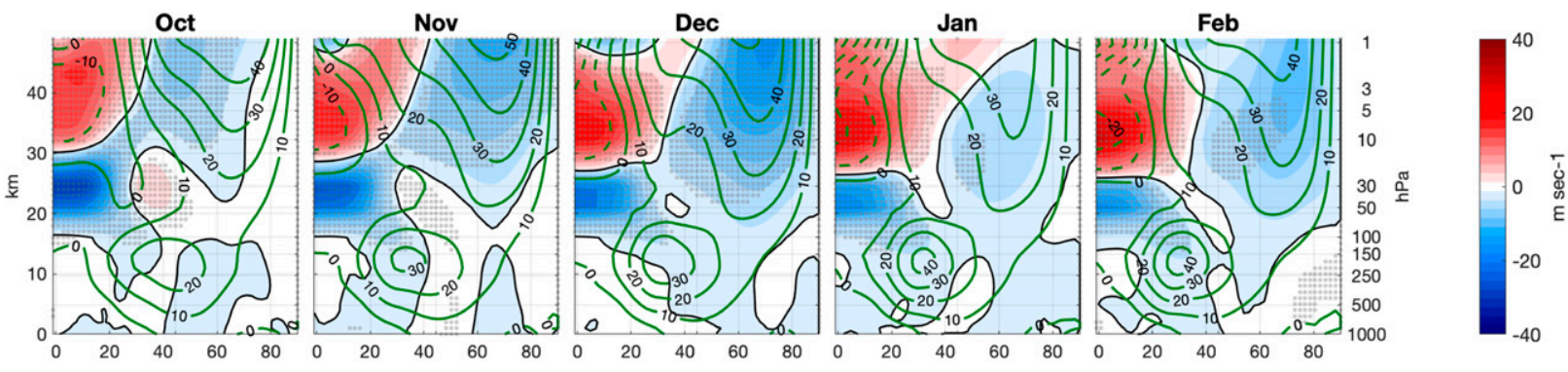

(a) $\bar{u}, \mathrm{E}-\mathrm{W} \mathrm{QBO}$
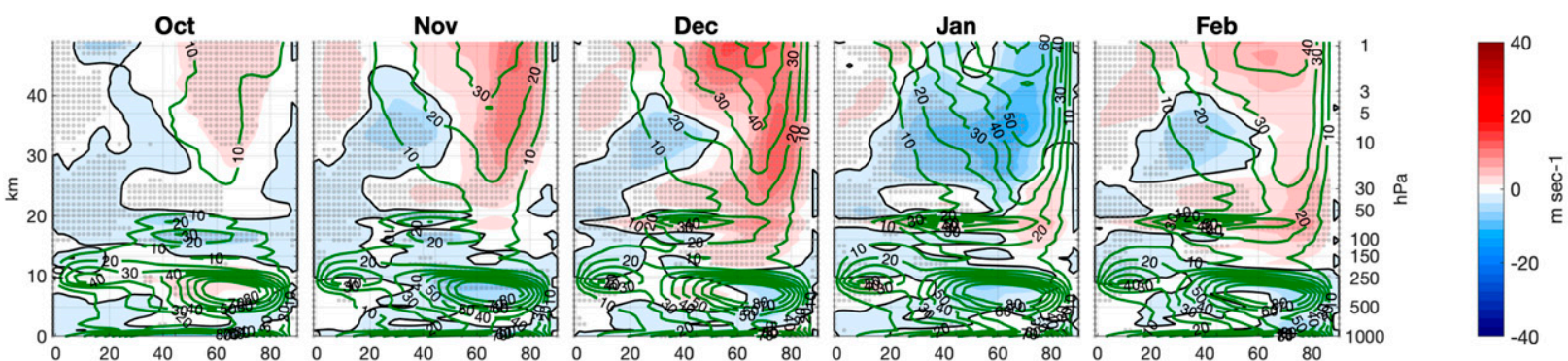

(b) $A^{*}, \mathrm{E}-\mathrm{W} \mathrm{QBO}$
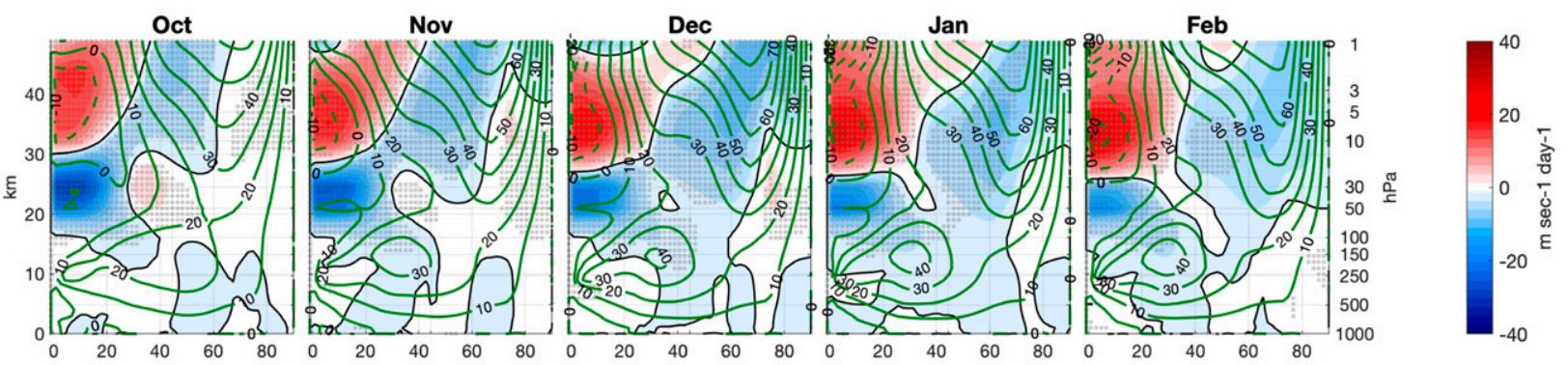

(c) $u_{r e f}, \mathrm{E}-\mathrm{W} \mathrm{QBO}$
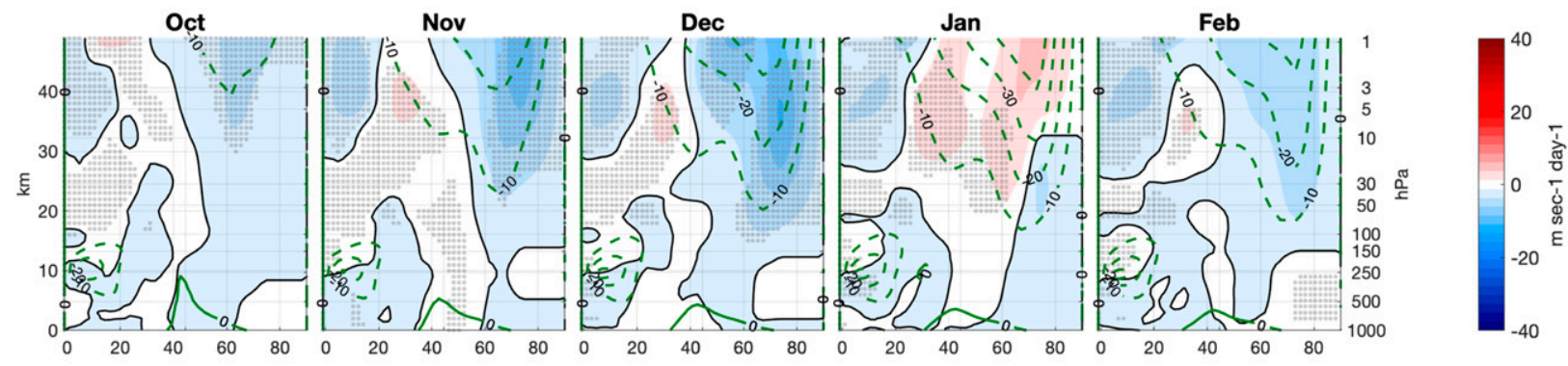

(d) $\Delta u, \mathrm{E}-\mathrm{W} \mathrm{QBO}$

FIG. 8. Monthly climatology of the east-west QBO difference (colors) and west QBO climatology (green contours) of the (a) zonalmean zonal wind $\left(\bar{u}, \mathrm{~m} \mathrm{~s}^{-1}\right)$, (b) $A^{*}\left(\mathrm{~m} \mathrm{~s}^{-1}\right)$, (c) zonal-mean reference state $\left(u_{\mathrm{ref}}, \mathrm{m} \mathrm{s}^{-1}\right)$, and (d) total zonal-mean zonal wind adjustment to the waves $\left(\Delta u, \mathrm{~m} \mathrm{~s}^{-1}\right)$, October-February, WACCM, 1955-2054. The dark highlighted contour denotes the zero line of the shaded values. Gray shading indicate statistically significant differences between the east and west QBO phases. 


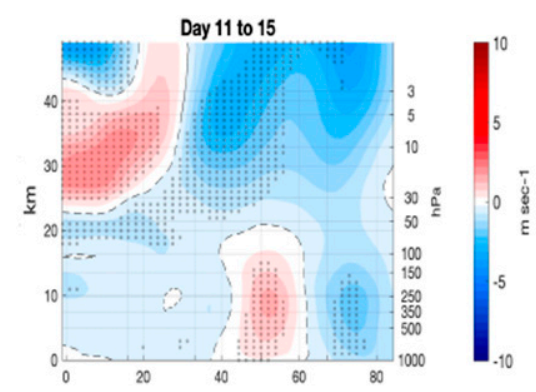

(a) $\mathrm{WACCM}, \bar{u}$, EQBO

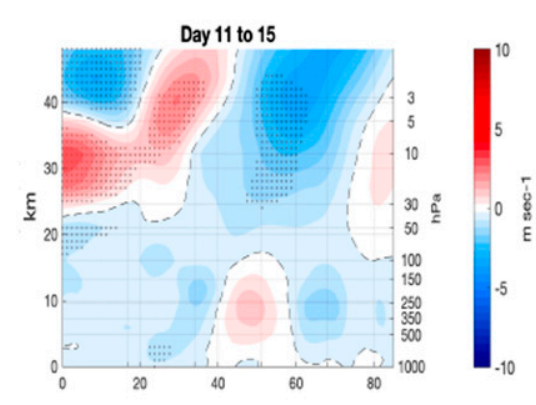

(d) JRA-55, $\bar{u}$, EQBO

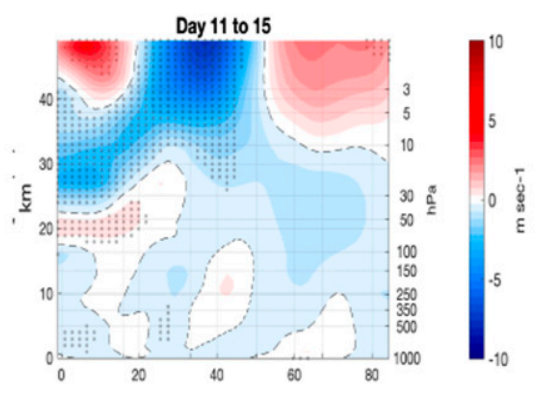

(b) $\mathrm{WACCM}, \bar{u}$, WQBO

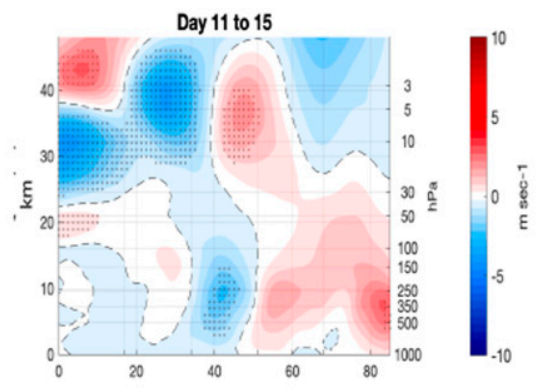

(e) JRA-55, $\bar{u}$, WQBO

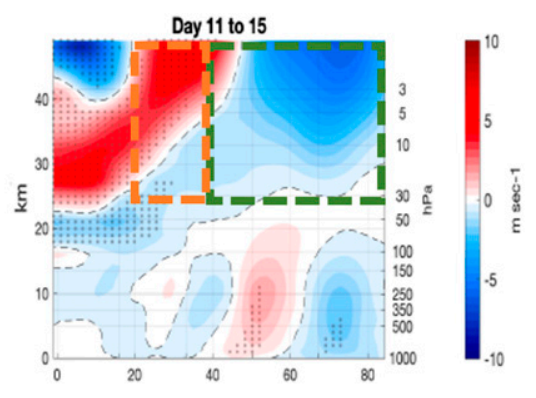

(c) WACCM, $\bar{u}$, E-W QBO

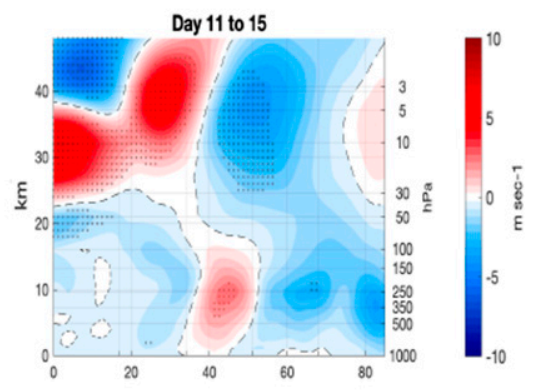

(f) JRA55, $\bar{u}$, E-W QBO

FIG. 9. Latitude-height composites of the zonal-mean zonal wind $\left(\bar{u}, \mathrm{~m} \mathrm{~s}^{-1}\right)$ at lag 11-15 days, showing anomalies from the climatology with respect to lag -5 days, for (left to right) east QBO, west QBO, and east-west QBO, showing (a)-(c) WACCM and (d)-(f) JRA-55, for the positive heat flux events (70th percentile of $\overline{v^{\prime} T^{\prime}}$ at $100 \mathrm{hPa}, 85^{\circ}-45^{\circ} \mathrm{N}$ ) during October. Gray shading indicates statistically significant differences between (a) and (b). The green and orange boxes in (c) show the area of averaging for the composites in Fig. 11.

between the datasets during west QBO, while they are quite similar during east QBO. Figure 10 shows the $A^{*}$ anomalies relative to day -5 , during west QBO years, for three phases of the wave life cycle, alongside the $11-15$ day $u_{\text {ref }}$ anomaly. The corresponding JRA-55 subplots from Fig. 7 are shown for reference. We see that for both datasets, at days 3-6 the $A^{*}$ anomaly extends throughout the mid-high-latitude stratosphere, and later on its peak shifts upward and equatorward, so that by days $11-15$, anomalies in the upper stratosphere are only significant at lower latitudes. In WACCM, however, $A^{*}$ spreads directly and smoothly to the subtropics whereas in JRA-55 there is a minimum in $A^{*}$ between $40^{\circ}$ and $50^{\circ} \mathrm{N}$ in the midstratosphere. More specifically, while in WACCM it appears that wave activity reaches the subtropical stratosphere by partial equatorward refraction of upward-propagating midlatitude waves. In JRA-55, it is possible that wave activity propagates to the subtropical stratosphere directly from the troposphere, or that it tunnels meridionally from a high-latitude waveguide through a subtropical evanescent region.This is consistent with there being qualitative differences in the index of refraction between JRA-55 and WACCM in the subtropical region. The relation between the wave geometry and wave structure at a given time is quite complicated (since it also depends on nonlocal wave sources and sinks), thus relating the minimum in $A^{*}$ to the wave geometry requires further study, and is not presented here. Regardless of the causes, it is clear that while in
WACCM we see a clear equatorward refraction of wave pulses during west QBO, in JRA-55, the evolution of $A^{*}$ during west QBO is more complex.

Despite these differences in wave propagation, their overall effect on the mean flow is quite similar between the two datasets. This is seen by looking at the day 11-15 $u_{\text {ref }}$ anomalies, which are plotted in Figs. $10 \mathrm{~b}$ and $10 \mathrm{~d}$. There is a significant region of deceleration at low latitudes (between $20^{\circ}$ and $50^{\circ} \mathrm{N}$ and between $20^{\circ}$ and $30^{\circ} \mathrm{N}$ for WACCM and JRA-55, respectively) which is mostly due to irreversible wave mixing. We also see significant acceleration immediately poleward of it (between $50^{\circ}$ and $70^{\circ} \mathrm{N}$ and between $30^{\circ}$ and $50^{\circ} \mathrm{N}$ for WACCM and JRA-55, respectively), likely due to sharpening of the PV gradients at the poleward edge of the mixing region.

To further quantify the reversibility of the flow, we repeat the analysis shown in Fig. 5. Figure 11 shows the composites of $\bar{u}, A^{*}, u_{\text {ref }}$, and $\Delta u$ anomalies relative to day -5 , for east and west QBO phases, in each of the two regions marked by the green and orange boxes in Fig. 9c. The main point this figure highlights is that despite the differences in wave propagation during west QBO, and in the location of acceleration and deceleration centers between WACCM and JRA-55, the qualitative behavior of the wave-induced deceleration hold. Namely, in the highlatitude box, the wave-induced deceleration only partially reverses, leaving a significant irreversible deceleration due to nonconservative processes. During west QBO, on the other 

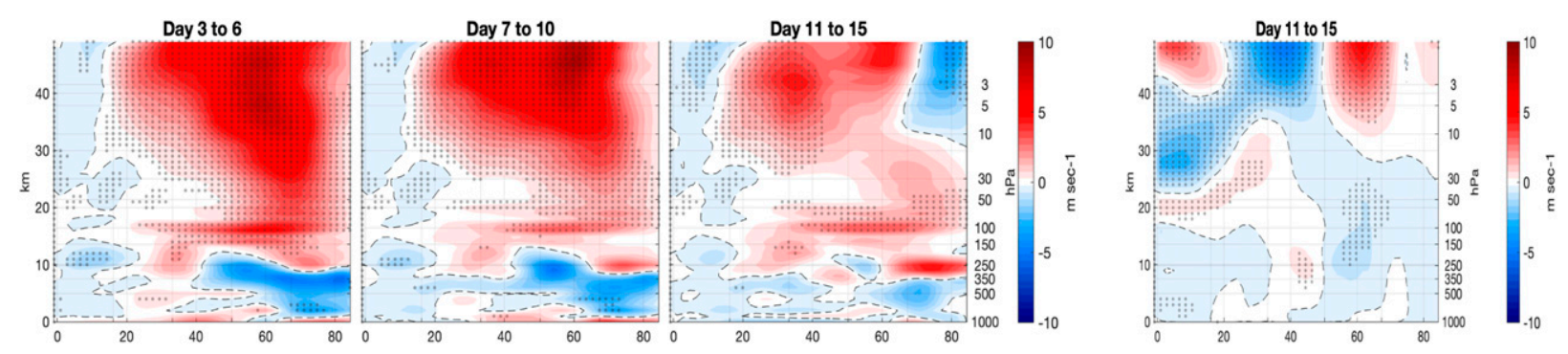

(a) $A^{*}$, WQBO, WACCM

(b) $u_{r e f}, \mathrm{WQBO}, \mathrm{WACCM}$
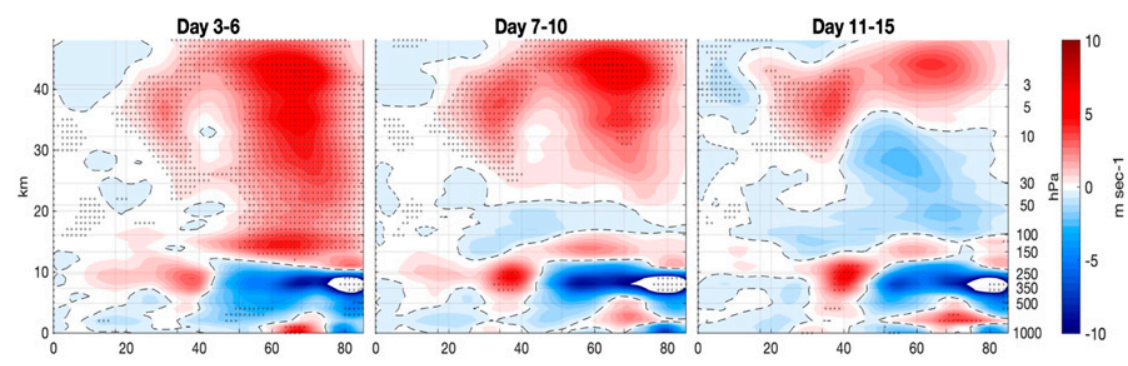

(c) $A^{*}$, WQBO, JRA-55

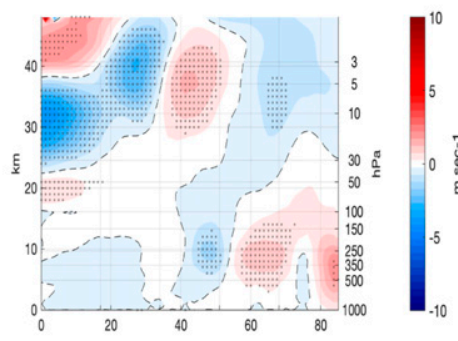

(d) $u_{r e f}$, WQBO, JRA-55

FIG. 10. Latitude-height time-lag composites of the (left) FAWA ( $\mathrm{m} \mathrm{s}^{-1}$ ) (lags 3-15) and (right) $u_{\text {ref }}$ (lag 11-15) for (top) WACCM and (bottom) JRA-55, showing anomalies from the climatology with respect to anomalies in lag -5 days, for west QBO events (positive heat flux events defined by the 70th percentile of $\overline{v^{\prime} T^{\prime}}$ at $100 \mathrm{hPa}, 85^{\circ}-45^{\circ} \mathrm{N}$ ), during October. Gray shading indicates statistically significant anomalies with respect to the climatology.

hand, the wave-induced deceleration fully reverses, consistent with the nonconservative processes being zero. In the lowlatitude box, as seen in JRA-55, during east QBO, the deceleration is weak and reversible (arising mostly from changes in $\Delta u$ ), while during west QBO, the wave-induced deceleration does not fully reverse, due to a strong and significant contribution from nonconservative processes $\left(u_{\text {ref }}\right)$, alongside a contribution from the conservative wave-induced deceleration $(\Delta u)$. Unlike for JRA-55 west QBO composite, the easterly anomaly in $\bar{u}$ does reach a peak and starts diminishing at day 12 , due to the $\Delta u$ anomaly decreasing after day 10 . This behavior is consistent with the cleaner equatorward refraction of waves in WACCM compared to JRA-55 during west QBO.

Silverman et al. (2018) showed that the differences in wave propagation between east and west QBO events, are consistent with the wave geometry of the vortex on which the wave events occur. ${ }^{3}$ Figure 12 shows the east minus west QBO composites of the Index of refraction squared (for zonal wavenumber 1 ) before the wave event starts (averaged over days -10 to -5$)$. The WACCM plot was shown in Silverman et al. (2018), and is shown here for comparison. We see a clear stronger $N_{\text {ref }}^{2}$ during east QBO poleward of $50^{\circ} \mathrm{N}$ and above 5hPA, and a weaker $N_{\text {ref }}^{2}$ at lower latitudes above $10 \mathrm{hPa}$, consistent with the waves refracting more toward the upper high-latitude vortex during east QBO, and more equatorward

\footnotetext{
${ }^{3}$ While the relation between the wave geometry and wave structure at a given time is quite complicated, anomalies in these quantities are often clearly related.
}

during west QBO. In JRA-55, we also see a significant reduction of $N_{\text {ref }}^{2}$ in the subtropics (between $20^{\circ}$ and $35^{\circ} \mathrm{N}, 30$ $3 \mathrm{hPa}$ ), consistent with a tendency of waves to be refracted equatorward during west QBO, but the region in which $N_{\text {ref }}^{2}$ looks different compared to WACCM-it is split into two separate regions above $5 \mathrm{hPa}$, centered around $30^{\circ}$ and $60^{\circ} \mathrm{N}$. This is consistent with the $\bar{u}$ and $A^{*}$ anomalies being latitudinally squeezed and shifted equatorward in JRA compared to WACCM.

\section{Conclusions}

The midlatitude QBO signal (the Holton-Tan effect) has been studied since its discovery for three decades, with most suggested mechanisms involving a modulation of planetary wave propagation and dissipation. However, most studies concentrated on the differences in wave propagation during midwinter, using monthly climatologies or models with perpetual winter conditions. This study complements previous studies by examining the onset of the QBO signal during fall (October) and by explicitly examining the effect of the QBO on the evolution of individual upward wave pulses and their effect on the zonal mean flow using a finiteamplitude wave activity diagnostic. Our analysis was performed on the long-term reanalysis record of JRA-55, and on a free-running WACCM simulation with a nudged QBO.

The following key results are found:

- In JRA-55, the midlatitude QBO signal of a weaker polar vortex during the east QBO phase starts in October. This signal strengthens until January when it begins to weaken. 


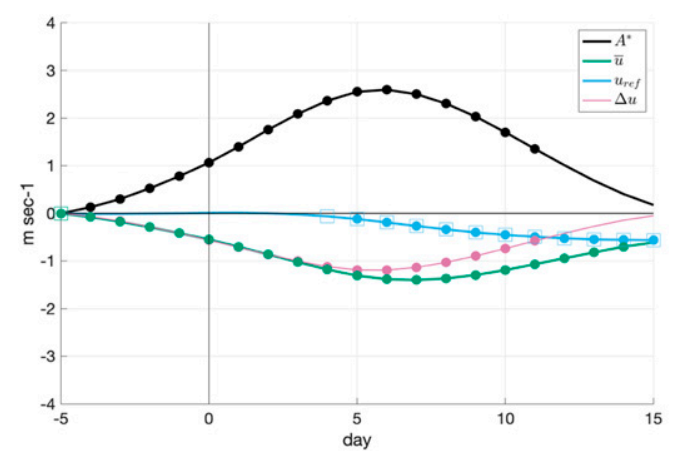

(a) $\mathrm{EQBO}, 85-40 \mathrm{~N}$

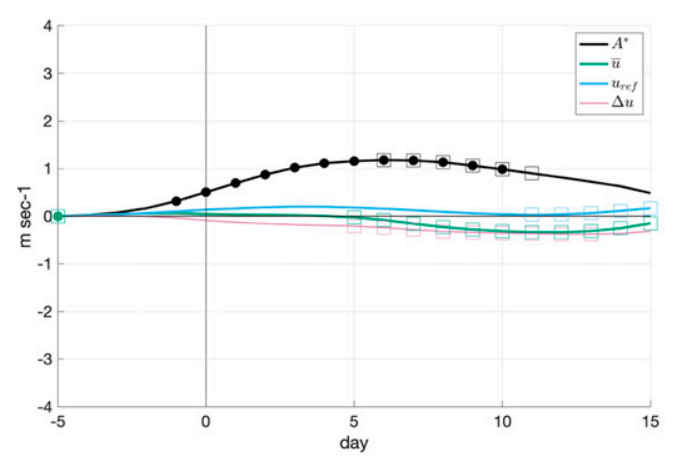

(c) EQBO, $20-40 \mathrm{~N}$

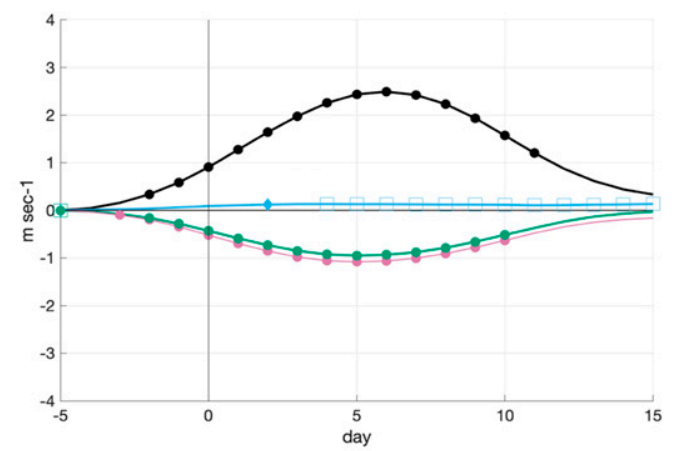

(b) WQBO, $85-40 \mathrm{~N}$

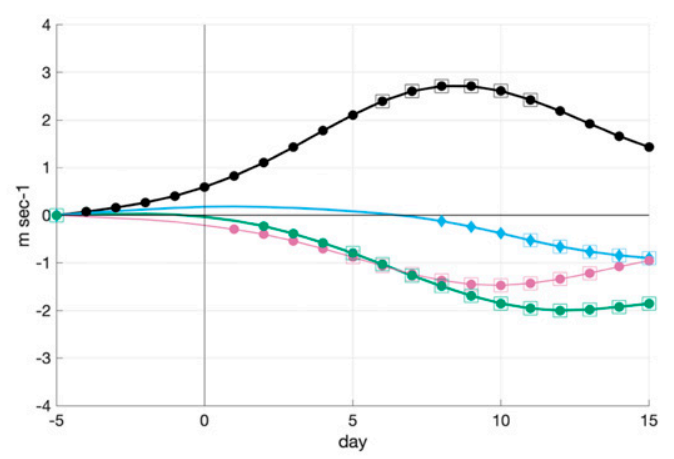

(d) WQBO, 20-40N

FIG. 11. Time-lag composites for the accumulated change (anomalies with respect to lag -5 days) of the zonalmean zonal wind $\bar{u}$ (green), $A^{*}$ (black), $u_{\text {ref }}$ (the change in winds due to nonconservative processes, blue), and $\Delta u$ (the adiabatic contribution to the wind tendency, pink). Averaged over $30-1 \mathrm{hPa}$, (top) $85^{\circ}-40^{\circ} \mathrm{N}$ and (bottom) $40^{\circ}-$ $20^{\circ} \mathrm{N}$, for upward wave pulse events during October for (left) east QBO and (right) west QBO, WACCM. Statistically significant values relative to the climatology are indicated by filled circles. Statistically significant differences between east and west QBO life cycles are marked by empty squares.

These changes in the polar vortex are explained by the differences in stratospheric wave activity between the two QBO phases (Fig. 2).

- Analysis of the life cycle of upward wave events during October shows a difference in the reversibility of the wave induced deceleration, despite the magnitude of the wave pulses being similar between the two QBO phases (Figs. 3, 4).

- During east QBO, the wave induced deceleration is irreversible in the main vortex region (between $40^{\circ}$ and $60^{\circ} \mathrm{N}$ ) and reversible to the south $\left(20^{\circ}-40^{\circ} \mathrm{N}\right)$. During west $\mathrm{QBO}$, on the other hand, the wave induced deceleration is reversible in the main vortex region and irreversible to the south (between $20^{\circ}$ and $40^{\circ} \mathrm{N}$ ). Correspondingly, the significant nonconservative mixing and diabatic processes occur between $60^{\circ}$ and $40^{\circ} \mathrm{N}$ during east QBO, and between $20^{\circ}$ and $40^{\circ} \mathrm{N}$ during west QBO (Figs. 5, 6). During both QBO phases, the wave induced deceleration is reversible poleward of $60^{\circ} \mathrm{N}$.

- Compared to JRA-55, in WACCM the strongest Holton-Tan signal (a weaker polar vortex during east QBO) occurs a month earlier. This is consistent with the waves being overall stronger and the vortex being weaker in WACCM. In addition, there are significant differences in the index of refraction at lower latitudes, which yield qualitative differences between the evolution of upward wave pulses when the QBO is in its westerly phase. These differences are manifest in the structure of the waves at lower latitudes, toward the end of their life cycle. Overall, these differences lead to the Holton-Tan signal in JRA-55 being a latitudinally compressed and slightly equatorward-shifted version of the signal in WACCM. Nonetheless, the underlying mechanism driving the Holton-Tan signal in both datasets is the change in the latitudinal location of the nonconservative wave mixing, and the reversibility of the wave induced deceleration.

In addition to the key findings in the stratosphere, we also found a weak but significant response of the tropospheric subtropical jet to the upward-propagating wave pulses, which results in a stronger and slightly poleward shifted tropospheric jet during east QBO. A similar tropospheric difference is found between the November climatologies of east and west QBO phases. These results hint to the existence of a QBO dependent mechanism that influences wave propagation in the troposphere. In this context, previous studies have shown that the 


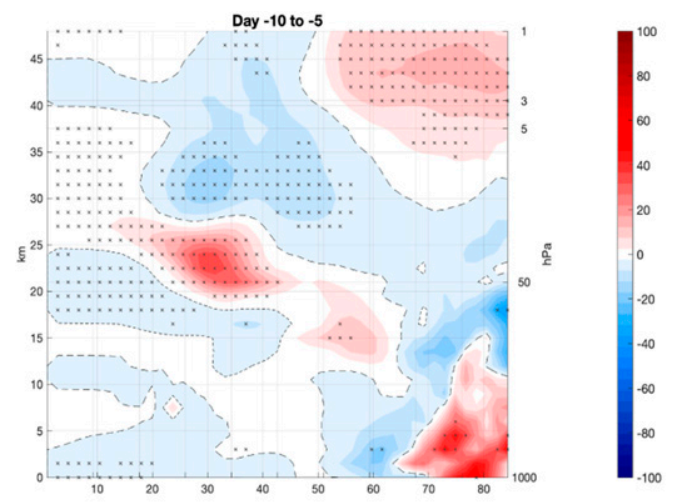

(a) $n,-10$ to -5 days, WACCM

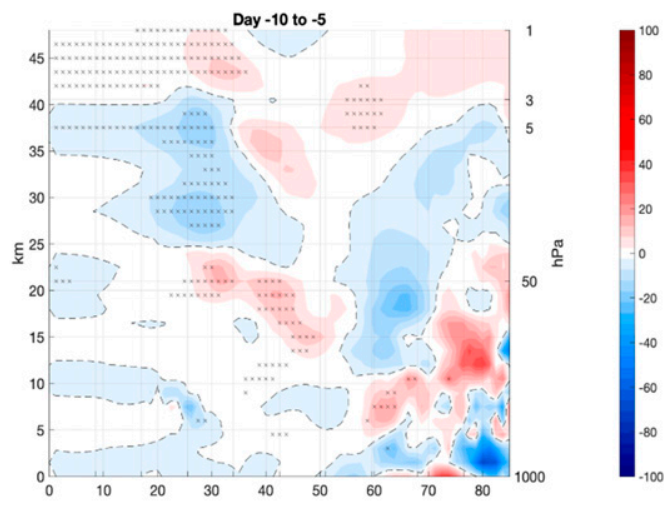

(b) $n,-10$ to -5 days, JRA-55

FIG. 12. Latitude-height of the index of refraction (for zonal wave 1) east and west QBO differences for (right) JRA-55 and (left) WACCM, during days -10 to -5 . Gray markers indicates statistically significant differences between east and west QBO.

stratospheric winter polar jet can modulate the structure of the tropospheric jet, by modulation of the baroclinic life cycles in the troposphere (e.g., Kunz et al. 2009; Rupp and Birner 2021). In principle, a modulation of the subtropical jet can further modulate the wave activity entering the stratosphere from below, acting as a feedback to the strengthen the development of the Holton-Tan effect which accumulates over fall, but a more focused examination of the underlying mechanisms controlling these feedbacks is needed to understand this troposphere-stratosphere coupling.

The seasonal development of the Holton-Tan signal is consistent with previous studies, which have suggested the modulation of planetary wave propagation is responsible for the weaker polar vortex during October-January of east QBO years (Garfinkel et al. 2012; Watson and Gray 2014; White et al. 2016; Lu et al. 2020; Dunkerton and Baldwin 1991; Hansen et al. 2013). The novelty of our analysis is in using the FAWA framework to explicitly diagnose the effects of the waves and nonconservative processes on the mean flow, and in examining how this process accumulates over individual upward-propagating wave events during October, when waves just start propagating up to the stratosphere on the newly formed polar vortex. This yields a mechanistic picture of the onset of the Holton-Tan signal. The examination of the QBO influence on individual upward wave events during October has been done by Silverman et al. (2018), but they used a small-amplitude enstrophy budget diagnostic which suffered from very large residuals, and they examined WACCM simulations. Our analysis extends this to the JRA55 dataset, and uses the FAWA diagnostic.

The FAWA diagnostic is related to the differences between an actual PV contour and its equivalent latitude, compared to enstrophy, which is related to deviations of the PV field from a zonal Eulerian mean. The use of a contour-based diagnostic, which applies to finite-amplitude waves, is advantageous also when the waves are small, because it directly diagnoses cases when the waves are completely conservative, meaning the PV contours return to their circular shape once the waves have left the domain, with no net change to the PV field. In this case, we expect the waves to have a zero net effect on the mean flow, as implied by the nonacceleration theorem for the case of conservative, statistically steady waves. The application of the nonacceleration concept to individual wave life cycles involves examining the overall effect that the waves had on the domain throughout the entire life cycle. As waves enter the stratosphere from the mid- or high-latitude troposphere below, they decelerate the flow simply by entering the domain and causing the PV contours to undulate (to satisfy Kelvin's circulation theorem; cf. Vallis 2017). By the end of the life cycle, the PV contours return to their circular shape. If the waves simply propagate up along the vortex and then equatorward to the subtropics, without any processes damping or enhancing them, the wave induced deceleration at the leading edge of the wave packet (where wave activity locally increases with time due to the approach of the wave) should be fully reversed by the wave-induced acceleration at the trailing edge of the wave packet (where the wave activity locally decreases with time due to the waves moving away). There are, however, some subtleties. To keep the flow in thermal wind balance, the wave-induced deceleration will also excite an overturning circulation which will partially counteract it, and also change the temperature gradient. Correspondingly, the trailing edge acceleration will excite an oppositely sensed overturning circulation which will fully reverse the changes it induces both on the zonal mean flow and on the temperature field. Theoretically, for statistically steady conservative waves the mean overturning circulation should vanish; however, the applicability to transient wave life cycles needs to be further examined. Our findings that the wave-induced deceleration over the main vortex region is fully reversible during October upward wave pulses when the QBO is in its westerly phase suggests the overturning circulation does indeed fully reverse over the wave life cycle. Lubis et al. (2016) also found a reversibility of the wave induced acceleration and the overturning circulation for downward-propagating wave events during midwinter. 
Another novelty of our analysis is the decomposition of the polar vortex deceleration and acceleration into contributions from conservative $(\Delta u)$ and nonconservative $\left(u_{\text {ref }}\right)$ processes. This allows an explicit calculation of the contribution of nonconservative processes with respect to an eddy free reference state, to the midlatitude QBO effect. A main finding is that the adiabatic response to the waves is dominant at high latitudes, while the influence of nonconservative processes on the waves and the mean flow are dominant at lower latitudes (in the surf zone region, where waves break). In the context of individual wave pulses, the contribution of nonconservative processes to the momentum and FAWA time tendencies [Eqs. (4) and (5)], can be further broken up to contributions from irreversible mixing acting on the waves or on the mean flow, and from diabatic wave processes (Fig. 6). Interestingly, we find regions in which nonconservative processes accelerate the vortex during an upward wave life cycle (e.g., Fig. 10b). Such an acceleration happens at the edge of the region of significant irreversible wave mixing, and most likely results from the sharpening of PV gradients at the edge of the mixing region. Lu et al. (2020) found a similar acceleration at the edge of the enhanced wave breaking region during west QBO later on in winter (November-December).

The monthly climatology of $u_{\text {ref }}$ shows regions in which the east-west QBO anomaly is positive, while the corresponding $\bar{u}$ anomaly is negative. This suggests changes in diabatic cooling of the polar cap during polar night also plays a role in modulating the midlatitude QBO signal (e.g., the positive $u_{\text {ref }}$ anomaly over the pole during December JRA-55, Fig. 2). This role of diabatic processes, has, to our knowledge, not been explicitly noted for the midlatitude effect of the QBO. Essentially, it is similar to a diabatic modulation of the polar night jet oscillation, pointed out by previous studies (e.g., Kuroda and Kodera 2001). A similar accelerating role for diabatic processes is also found in the recovery stage of sudden stratospheric warmings (Hitchcock and Shepherd 2013; Lubis et al. 2018b; Kwasniok et al. 2019).

Understanding the effect of individual wave pulses on the deceleration of the vortex, allows us to mechanistically understand the effect of other physical processes on the vortex, and their interaction with the QBO. For example, in Silverman et al. (2018) we showed how interactive ozone waves modify individual upward wave life cycles by changing the thermal damping of the waves. We further found that the overall effect this change in thermal damping has on the waves differs between east and west QBO, because of the qualitatively different nature of the wave life cycles between the QBO phases. In a similar manner, this framework can be used to study the effect of other processes, like solar variations, and how their influence changes depending on the phase of the QBO, and more generally, on the life cycle of individual wave events, and the morphology of their dissipation.

Acknowledgments. This work is supported by the GermanIsraeli Foundation for Scientific Research and Development, under Grant GIF1151-83.8/2011, and by the Israel Science
Foundation (Grants 1537/12 and 1685/17). This work has also been partially performed within the Helmholtz University Young Investigators Group NATHAN funded by the Helmholtz Association through the President's Initiative and Networking Fund and the GEOMAR-Helmholtz Centre for Ocean Research Kiel, Germany. We also thank Sebastian Wahl for his assistance in running the WACCM simulation. The model simulations were performed at the German Climate Computing Centre [Deutsches Klimarechenzentrum (DKRZ)] in Hamburg, Germany. We acknowledge the Israeli Atmospheric and Climatic Data Center, supported by the Israeli Ministry of Science, Technology and Space, for assisting with the data handling. We thank the anonymous reviewers for their helpful comments.

Data availability statement. The CESM(WACCM) model data requests should be addressed to Katja Matthes (kmatthes@ geomar.de). JRA-55 data can be downloaded from Research Data Archive at the National Center for Atmospheric Research, Computational and Information Systems Lab (https://rda.ucar.edu; Japan Meteorological Agency 2013). Computation of the FAWA terms can be done with the Python package found at https:// github.com/csyhuang/hn2016_falwa.

\section{REFERENCES}

Allen, D. R., and N. Nakamura, 2003: Tracer equivalent latitude: A diagnostic tool for isentropic transport studies. J. Atmos. Sci., 60, 287-304, https://doi.org/10.1175/1520-0469(2003)060<0287: TELADT $>2.0 . \mathrm{CO} ; 2$.

Andrews, D. G., and M. E. McIntyre, 1976: Planetary waves in horizontal and vertical shear: The generalized Eliassen-Palm relation and the mean zonal acceleration. J. Atmos. Sci., 33, 2031-2048, https://doi.org/10.1175/1520-0469(1976)033<2031: PWIHAV $>2.0 . \mathrm{CO} ; 2$.

- C. B. Leovy, and J. R. Holton, 1987: Middle Atmosphere Dynamics. International Geophysics Series, Vol. 40, Academic Press, 489 pp.

Anstey, J. A., and T. G. Shepherd, 2014: High-latitude influence of the quasi-biennial oscillation. Quart. J. Roy. Meteor. Soc., 140, 1-21, https://doi.org/10.1002/qj.2132.

— oscillation in a multi-model ensemble of QBO-resolving models. Quart. J. Roy. Meteor. Soc., https://doi.org/10.1002/ qj. 4048 , in press.

Burrows, D. A., G. Chen, and L. Sun, 2017: Barotropic and baroclinic eddy feedbacks in the midlatitude jet variability and responses to climate change-like thermal forcings. J. Atmos. Sci., 74, 111-132, https://doi.org/10.1175/JAS-D-16-0047.1.

Butchart, N., and E. E. Remsberg, 1986: The area of the stratospheric polar vortex as a diagnostic for tracer transport on an isentropic surface. J. Atmos. Sci., 43, 1319-1339, https://doi.org/10.1175/ 1520-0469(1986)043<1319:TAOTSP>2.0.CO;2.

Charlton, A. J., and L. M. Polvani, 2007: A new look at stratospheric sudden warmings. Part I: Climatology and modeling benchmarks. J. Climate, 20, 449-469, https://doi.org/10.1175/ JCLI3996.1.

Charney, J., and P. Drazin, 1961: Propagation of planetary-scale disturbances from the lower into the upper atmosphere. J. Geophys. Res., 66, 83-109, https://doi.org/10.1029/JZ066i001p00083.

Dunkerton, T. J., and M. P. Baldwin, 1991: Quasi-biennial modulation of planetary-wave fluxes in the Northern Hemisphere 
winter. J. Atmos. Sci., 48, 1043-1061, https://doi.org/10.1175/ 1520-0469(1991)048< 1043:QBMOPW > 2.0.CO;2.

Dunn-Sigouin, E., and T. A. Shaw, 2015: Comparing and contrasting extreme stratospheric events, including their coupling to the tropospheric circulation. J. Geophys. Res. Atmos., 120, 1374-1390, https://doi.org/10.1002/2014JD022116.

Ebdon, R., 1975: The quasi-biennial oscillation and its association with tropospheric circulation pattern. Meteor. Mag., 104, 282-297.

Garfinkel, C. I., T. A. Shaw, D. L. Hartmann, and D. W. Waugh, 2012: Does the Holton-Tan mechanism explain how the quasibiennial oscillation modulates the Arctic polar vortex? J. Atmos. Sci., 69, 1713-1733, https://doi.org/10.1175/JAS-D-11-0209.1.

Gelaro, R., and Coauthors, 2017: The Modern-Era Retrospective Analysis for Research and Applications, version 2 (MERRA2). J. Climate, 30, 5419-5454, https://doi.org/10.1175/JCLI-D16-0758.1.

Gent, P. R., and Coauthors, 2011: The Community Climate System Model version 4. J. Climate, 24, 4973-4991, https://doi.org/ 10.1175/2011JCLI4083.1.

GMAO, 2015a: MERRA-2 M2I3NPASM: 3D, 3-hourly, instantaneous, pressure-level, assimilation, assimilated meteorological fields V5.12.4. GES DISC, accessed April 2015, https:// doi.org/10.5067/QBZ6MG944HW0.

— 2015b: MERRA-2 M2T3NPTDT: 3D, 3-hourly, timeaveraged, pressure-level, assimilation, temperature tendencies V5.12.4. GES DISC, accessed April 2015, https://doi.org/ 10.5067/9NCR9DDDOPFI0.

Gray, L. J., S. Crooks, C. Pascoe, S. Sparrow, and M. Palmer, 2004: Solar and QBO influences on the timing of stratospheric sudden warmings. J. Atmos. Sci., 61, 2777-2796, https:// doi.org/10.1175/JAS-3297.1.

Hansen, F., K. Matthes, and L. Gray, 2013: Sensitivity of stratospheric dynamics and chemistry to QBO nudging width in the chemistry-climate model WACCM. J. Geophys. Res. Atmos., 118, 10-464, https://doi.org/10.1002/jgrd.50812.

Harnik, N., 2009: Observed stratospheric downward reflection and its relation to upward pulses of wave activity. J. Geophys. Res., 114, D08120, https://doi.org/10.1029/2008JD010493.

Haynes, P. H., 1988: Forced, dissipative generalizations of finiteamplitude wave-activity conservation relations for zonal and nonzonal basic flows. J. Atmos. Sci., 45, 2352-2362, https:// doi.org/10.1175/1520-0469(1988)045<2352:FDGOFA>2.0.CO;2.

Hitchcock, P., and T. G. Shepherd, 2013: Zonal-mean dynamics of extended recoveries from stratospheric sudden warmings. J. Atmos. Sci., 70, 688-707, https://doi.org/10.1175/JAS-D-120111.1.

Holton, J. R., and H.-C. Tan, 1980: The influence of the equatorial quasi-biennial oscillation on the global circulation at $50 \mathrm{mb}$. J. Atmos. Sci., 37, 2200-2208, https://doi.org/10.1175/15200469(1980)037<2200:TIOTEQ>2.0.CO;2.

$\mathrm{Hu}, \mathrm{Y}$., and K. K. Tung, 2002: Tropospheric and equatorial influences on planetary-wave amplitude in the stratosphere. Geophys. Res. Lett., 29, 1019, https://doi.org/10.1029/2001GL013762.

Huang, C. S., and N. Nakamura, 2016: Local finite-amplitude wave activity as a diagnostic of anomalous weather events. J. Atmos. Sci., 73, 211-229, https://doi.org/10.1175/JAS-D15-0194.1.

_ and _ 2017: Local wave activity budgets of the wintertime Northern Hemisphere: Implication for the Pacific and Atlantic storm tracks. Geophys. Res. Lett., 44, 5673-5682, https:// doi.org/10.1002/2017GL073760.

Japan Meteorological Agency, 2013: JRA-55: Japanese 55-year Reanalysis, daily 3-hourly and 6-hourly data. NCAR Research
Data Archive Computational and Information Systems Laboratory, accessed 1 April 2015, https://doi.org/10.5065/ D6HH6H41.

Killworth, P. D., and M. E. McIntyre, 1985: Do Rossby-wave critical layers absorb, reflect, or over-reflect? J. Fluid Mech., 161, 449-492, https://doi.org/10.1017/S0022112085003019.

Kobayashi, S., and Coauthors, 2015: The JRA-55 Reanalysis: General specifications and basic characteristics. J. Meteor. Soc. Japan, 93, 5-48, https://doi.org/10.2151/jmsj.2015-001.

Kunz, T., K. Fraedrich, and F. Lunkeit, 2009: Response of idealized baroclinic wave life cycles to stratospheric flow conditions. J. Atmos. Sci., 66, 2288-2302, https://doi.org/10.1175/2009JAS2827.1.

Kuroda, Y., and K. Kodera, 2001: Variability of the polar night jet in the Northern and Southern Hemispheres. J. Geophys. Res., 106, 20 703-20 713, https://doi.org/10.1029/2001JD900226.

Kwasniok, F., R. Beaumont, and J. Thuburn, 2019: Vortex dynamics of stratospheric sudden warmings: A reanalysis data study using PV contour integral diagnostics. Quart. J. Roy. Meteor. Soc., 145, 1013-1033, https://doi.org/10.1002/qj.3474.

Lean, J., G. Rottman, J. Harder, and G. Kopp, 2005: SORCE contributions to new understanding of global change and solar variability. The Solar Radiation and Climate Experiment (SORCE): Mission Description and Early Results, G. Rottman, T. Woods, and V. George, Eds., Springer, 27-53.

Li, K.-F., and K.-K. Tung, 2014: Quasi-biennial oscillation and solar cycle influences on winter Arctic total ozone. J. Geophys. Res. Atmos., 119, 5823-5835, https://doi.org/10.1002/2013JD021065.

Lu, H., M. P. Baldwin, L. J. Gray, and M. J. Jarvis, 2008: Decadalscale changes in the effect of the QBO on the northern stratospheric polar vortex. J. Geophys. Res., 113, D10114, https://doi.org/10.1029/2007JD009647.

- M. H. Hitchman, L. J. Gray, J. A. Anstey, and S. M. Osprey, 2020: On the role of Rossby wave breaking in the quasibiennial modulation of the stratospheric polar vortex during boreal winter. Quart. J. Roy. Meteor. Soc., 146, 1939-1959, https://doi.org/10.1002/qj.3775.

Lubis, S. W., K. Matthes, N.-E. Omrani, N. Harnik, and S. Wahl, 2016: Influence of the quasi-biennial oscillation and sea surface temperature variability on downward wave coupling in the Northern Hemisphere. J. Atmos. Sci., 73, 1943-1965, https://doi.org/10.1175/JAS-D-15-0072.1.

_ V. Silverman, K. Matthes, N. Harnik, N.-E. Omrani, and S. Wahl, 2017: How does downward planetary wave coupling affect polar stratospheric ozone in the Arctic winter stratosphere? Atmos. Chem. Phys., 17, 2437-2458, https://doi.org/ 10.5194/acp-17-2437-2017.

- C. C. S. Huang, and N. Nakamura, 2018a: Role of finiteamplitude eddies and mixing in the life cycle of stratospheric sudden warmings. J. Atmos. Sci., 75, 3987-4003, https:// doi.org/10.1175/JAS-D-18-0138.1.

, N.-E. Omrani, and M. Jucker, 2018b: Role of finiteamplitude Rossby waves and nonconservative processes in downward migration of extratropical flow anomalies. J. Atmos. Sci., 75, 1385-1401, https://doi.org/10.1175/JAS-D-17-0376.1.

Marsh, D. R., M. J. Mills, D. E. Kinnison, J.-F. Lamarque, N. Calvo, and L. M. Polvani, 2013: Climate change from 1850 to 2005 simulated in CESM1(WACCM). J. Climate, 26, 7372-7391, https://doi.org/10.1175/JCLI-D-12-00558.1.

Matthes, K., D. R. Marsh, R. R. Garcia, D. E. Kinnison, F. Sassi, and S. Walters, 2010: Role of the QBO in modulating the influence of the 11 year solar cycle on the atmosphere using constant forcings. J. Geophys. Res., 115, D18110, https:// doi.org/10.1029/2009JD013020. 
McIntyre, M., and T. Shepherd, 1987: An exact local conservation theorem for finite-amplitude disturbances to non-parallel shear flows, with remarks on Hamiltonian structure and on Arnold's stability theorems. J. Fluid Mech., 181, 527-565, https://doi.org/10.1017/S0022112087002209.

Naito, Y., and I. Hirota, 1997: Interannual variability of the northern winter stratospheric circulation related to the QBO and the solar cycle. J. Meteor. Soc. Japan, 75, 925-937, https:// doi.org/10.2151/jmsj1965.75.4_925.

Nakamura, N., and A. Solomon, 2010: Finite-amplitude wave activity and mean flow adjustments in the atmospheric general circulation. Part I: Quasigeostrophic theory and analysis. J. Atmos. Sci., 67, 3967-3983, https://doi.org/10.1175/2010JAS3503.1.

_ , and D. Zhu, 2010: Finite-amplitude wave activity and diffusive flux of potential vorticity in eddy-mean flow interaction. J. Atmos. Sci., 67, 2701-2716, https://doi.org/10.1175/ 2010JAS3432.1.

— in the jet stream. Science, 361, 42-47, https://doi.org/10.1126/ science.aat 0721.

Perlwitz, J., and N. Harnik, 2003: Observational evidence of a stratospheric influence on the troposphere by planetary wave reflection. J. Climate, 16, 3011-3026, https://doi.org/10.1175/ 1520-0442(2003)016<3011:OEOASI $>2.0$. CO 2 .

Plumb, R. A., 1989: On the seasonal cycle of stratospheric planetary waves. Pure Appl. Geophys., 130, 233-242, https://doi.org/ 10.1007/BF00874457.

— 2002: Stratospheric transport. J. Meteor. Soc. Japan, 80, 793809, https://doi.org/10.2151/jmsj.80.793.

— on an equatorial beta-plane. Quart. J. Roy. Meteor. Soc., 108, 335-352, https://doi.org/10.1002/qj.49710845604.

Polvani, L. M., and D. W. Waugh, 2004: Upward wave activity flux as a precursor to extreme stratospheric events and subsequent anomalous surface weather regimes. J. Climate, 17, 3548-3554, https://doi.org/10.1175/1520-0442(2004)017<3548: UWAFAA $>2.0 . \mathrm{CO} ; 2$.
Reichler, T., J. Kim, E. Manzini, and J. Kröger, 2012: A stratospheric connection to Atlantic climate variability. Nat. Geosci., 5, 783787, https://doi.org/10.1038/ngeo1586.

Rupp, P., and T. Birner, 2021: Tropospheric eddy feedback to different stratospheric conditions in idealised baroclinic life cycles. Wea. Climate Dyn., 2, 111-128, https://doi.org/10.5194/ wcd-2-111-2021.

Ruzmaikin, A., J. Feynman, X. Jiang, and Y. Yung, 2005: Extratropical signature of the quasi-biennial oscillation. J. Geophys. Res., 110, D11111, https://doi.org/10.1029/2004JD005382.

Shaw, T. A., and J. Perlwitz, 2013: The life cycle of Northern Hemisphere downward wave coupling between the stratosphere and troposphere. J. Climate, 26, 1745-1763, https:// doi.org/10.1175/JCLI-D-12-00251.1.

Shuckburgh, E., W. Norton, A. Iwi, and P. Haynes, 2001: Influence of the quasi-biennial oscillation on isentropic transport and mixing in the tropics and subtropics. J. Geophys. Res., 106, 14327-14 337, https://doi.org/10.1029/2000JD900664.

Silverman, V., N. Harnik, K. Matthes, S. W. Lubis, and S. Wahl, 2018: Radiative effects of ozone waves on the Northern Hemisphere polar vortex and its modulation by the QBO. Atmos. Chem. Phys., 18, 6637-6659, https://doi.org/10.5194/ acp-18-6637-2018.

Solomon, A., and N. Nakamura, 2012: An exact Lagrangian-mean wave activity for finite-amplitude disturbances to barotropic flow on a sphere. J. Fluid Mech., 693, 69-92, https://doi.org/ 10.1017/jfm.2011.460.

Vallis, G. K., 2017: Atmospheric and Oceanic Fluid Dynamics. Cambridge University Press, 946 pp.

Watson, P. A., and L. J. Gray, 2014: How does the quasi-biennial oscillation affect the stratospheric polar vortex? J. Atmos. Sci., 71, 391-409, https://doi.org/10.1175/JAS-D-13-096.1.

White, I. P., H. Lu, and N. J. Mitchell, 2016: Seasonal evolution of the QBO-induced wave forcing and circulation anomalies in the northern winter stratosphere. J. Geophys. Res. Atmos., 121, $10411-10431$, https://doi.org/10.1002/ 2015JD024507. 